\title{
Fluctuations Analysis of Finite Discrete Birth and Death Chains with Emphasis on Moran Models with Mutations
}

\author{
Thierry E. Huillet \\ Laboratoire de Physique Théorique et Modélisation, CNRS-UMR 8089 et Université de Cergy-Pontoise, 2 avenue Adolphe Chauvin, \\ 95302 Cergy-Pontoise, France \\ Correspondence should be addressed to Thierry E. Huillet; huillet@u-cergy.fr
}

Received 27 May 2013; Accepted 2 July 2013

Academic Editors: E. M. Cherry, B. Foy, and M. Glavinovic

Copyright (C) 2013 Thierry E. Huillet. This is an open access article distributed under the Creative Commons Attribution License, which permits unrestricted use, distribution, and reproduction in any medium, provided the original work is properly cited.

The Moran model is a discrete-time birth and death Markov chain describing the evolution of the number of type 1 alleles in a haploid population with two alleles whose total size $N$ is preserved during the course of evolution. Bias mechanisms such as mutations or selection can affect its neutral dynamics. For the ergodic Moran model with mutations, we get interested in the fixation probabilities of a mutant, the growth rate of fluctuations, the first hitting time of the equilibrium state starting from state $\{0\}$, the first return time to the equilibrium state, and the first hitting time of $\{N\}$ starting from $\{0\}$, together with the time needed for the walker to reach its invariant measure, again starting from $\{0\}$. For the last point, an appeal to the notion of Siegmund duality is necessary, and a cutoff phenomenon will be made explicit. We are interested in these problems in the large population size limit $N \rightarrow \infty$. The Moran model with mutations includes the heat exchange models of Ehrenfest and Bernoulli-Laplace as particular cases; these were studied from the point of view of the controversy concerning irreversibility ( $H$-theorem) and the recurrence of states.

\section{Introduction}

In Section 2, we start with generalities on discrete-time birth and death Markov chains with finite state space, whose transition matrix is of Jacobi type: spectral representation, reversibility versus $H$-theorem, large deviations of the empirical average, invariant measure, conditions of transience/recurrence, spectral positivity and stochastic monotonicity, distribution of hitting and first return times, and Greens function. The scale or harmonic function is shown to be of interest to determine the excursions heights would the chain be ergodic. After having fixed the background, we then proceed with the study of specific Moran chains presenting various bias mechanisms.

The Moran chain model is a particular instance of a discrete-time birth and death Markov chain, describing the evolution of the number of type 1 alleles in an haploid population with two alleles whose total size $N$ is preserved during the course of evolution. Bias mechanisms such as mutations or selection can affect its neutral dynamics (there are alternative domains of science where this model makes sense, namely, economy and behavioral sciences. Here, the Markov state $X_{n}$ takes on the interpretation of the number of agents that belong to the first strategy 1 (say right- or leftwing voters) at time $n$. See $[1,2]$ and references therein for a recent study with this physical image in mind.).

For the ergodic Moran model with mutations [3], we get interested (in Sections 3 and 5) into the fixation probabilities of a mutant, the growth of fluctuations, the first hitting time of the equilibrium state starting from $\{0\}$, the first return time to the equilibrium state, and the first hitting time of $\{N\}$ starting from $\{0\}$, together with the time needed for the Moran walker to reach its invariant measure, again starting from $\{0\}$. The detailed growth rates of these quantities are shown to be exactly determined in terms of the mutation probabilities.

(i) For the first fixation probability point, a sharp threshold phenomenon is exhibited in the following sense: would the mutation probability favoring allele 1 exceed the one favoring allele 2 , the fixation probability of a type 1 mutant tends to 1 as $N$ grows large with a $\mathcal{O}(1 / N)$ correction. On the contrary, this fixation 
probability tends to 0 geometrically fast as $N$ grows large. In the critical case when the two mutation probabilities are egal, this fixation probability goes to $1 / 2$ from below.

Under the additional presence of selection, the fixation condition can be achieved with probability 1 even if the mutation probability favoring allele 2 exceeds the one favoring allele 1 . The condition is that the relative fitness of allele 1 should be large enough with bound given in terms of a ratio of mutation probabilities, translating a mutation-selection balance.

(ii) For the last time to stationarity point, an appeal to the notion of Siegmund duality (requiring stochastic monotonicity) is necessary, and a cut-off phenomenon will be made explicit in Section 4, following the general stream of ideas developed in [4-7]. The analysis is made simple because the spectral representation of the mutation Moran model is exactly known.

We are interested into these problems in the large population size limit $N \rightarrow \infty$ (i.e., in the thermodynamic limit). From our analysis, it follows that the mutation Moran walker started at $\{0\}$ reaches stationarity in about $\mathcal{O}(N \log N)$ time, far below the time needed to first return to the origin (or excursion's length) which is geometrically large with $N$. This is because the mutational drift pushes the walker inside the bulk of the state space, making the reverse move to any of the two boundaries extremely costly.

\section{Discrete-Time Birth and Death Processes}

We start with generalities on discrete birth and death (BD) processes with finite state space, before particularizing the study to the Moran model under concern which is in this class.

2.1. Generalities on BD Processes with Finite State Space. Let $P$ be a $(N+1)^{2}$ tridiagonal (Jacobi) irreducible stochastic matrix

$$
P=\left[\begin{array}{ccccc}
r_{0} & p_{0} & & & \\
q_{1} & r_{1} & p_{1} & & \\
& \ddots & \ddots & \ddots & \\
& & q_{N-1} & r_{N-1} & p_{N-1} \\
& & & q_{N} & r_{N}
\end{array}\right]
$$

with $p_{0}>0, q_{x}, p_{x}>0, x=1, \ldots, N-1$, and $q_{N}>0$, corresponding to the transition probability matrix of some ergodic discrete-time Markov chain $X_{n}$ on the state space $\{0, \ldots, N\}$. With 1 a column vector of $1 \mathrm{~s}, P$ is stochastic if $P \mathbf{1}=\mathbf{1}$.

The left (row-) eigenvector $\pi^{\prime}:=\left(\pi_{0}, \pi_{1}, \ldots, \pi_{N}\right)$ associated to the eigenvalue 1 satisfies $\pi^{\prime}=\pi^{\prime} P$, where ${ }^{\prime}$ denotes transposition. Its components are given by $\pi_{0}$ and $\pi_{y}=\pi_{0} \prod_{z=0}^{y-1}\left(p_{z} / q_{z+1}\right)>0, y=1, \ldots, N$, with $\pi_{0}$ chosen so as $\sum_{y=0}^{N} \pi_{y}=1$. It corresponds to the invariant probability measure of the chain.
The matrix $P$ is diagonally equivalent to the symmetric matrix

$$
P_{S}=\left[\begin{array}{ccccc}
r_{0} & \sqrt{p_{0} q_{1}} & & & \\
\sqrt{p_{0} q_{1}} & r_{1} & \sqrt{p_{1} q_{2}} & & \\
& \ddots & \ddots & \ddots & \\
& & \sqrt{p_{N-2} q_{N-1}} & r_{N-1} & \sqrt{p_{N-1} q_{N}} \\
& & & \sqrt{p_{N-1} q_{N}} & r_{N}
\end{array}\right],
$$

so with real eigenvalues. Indeed, with $D_{\pi}:=\operatorname{diag}\left(\pi_{0}, \ldots, \pi_{N}\right)$, we have $P=D_{\pi}^{-1 / 2} P_{S} D_{\pi}^{1 / 2}$. As a symmetric matrix, $P_{S}$ is diagonalizable by an orthogonal transformation, and so, $P$ is diagonalizable.

Such nearest-neighbors random walks models are well known to be reversible (detailed balance holds). Indeed, $P=$ $\overleftarrow{P}$, where $\overleftarrow{P}$ is the transition matrix of the time-reversed process, given by $\overleftarrow{P}^{\prime}=D_{\pi} P D_{\pi}^{-1}$ (with $P^{\prime}$ denoting the transpose of $P$ ). The transition matrix of reversible Markov chains has real eigenvalues.

Under our assumptions, irreducible chains governed by $P$ are positive recurrent, meaning that every state recurs with probability 1 , the mean time between recurrence to state $x$ being finite and equal to $1 / \pi_{x}$.

2.2. The Karlin-McGregor (KMG) Spectral Theory. Consider the polynomials $\left(\mathfrak{q}_{y}(t) ; y=0, \ldots, N\right)$ in the variable $t \in$ $[-1,1]$, determined by $\mathfrak{q}_{0}(t)=1$ and the 3 -term recurrences:

$$
\begin{gathered}
t \mathfrak{q}_{0}(t)=p_{0} \mathfrak{q}_{1}(t)+r_{0} \mathfrak{q}_{0}(t), \\
t \mathfrak{q}_{y}(t)=p_{y} \mathfrak{q}_{y+1}(t)+r_{y} \mathfrak{q}_{y}(t) \\
\quad+q_{y} \mathfrak{q}_{y-1}(t), \quad y \in\{1, N-1\}
\end{gathered}
$$

therefore, with $\mathfrak{q}_{0}(t)=1, \mathfrak{q}_{1}(t)=\left(t-r_{0}\right) / p_{0}, \mathfrak{q}_{2}(t)=[(t-$ $\left.\left.r_{1}\right) \mathfrak{q}_{1}(t)-q_{1}\right] / p_{1}, \ldots$. The polynomials $\mathfrak{q}_{y}(t)$ satisfy $\mathfrak{q}_{y}(1)=$ $1, y \geq 0$ and are of degree $y$ in $t$. They are often called the random walk polynomials. Let $t_{k}, k=0, \ldots, N$ be the zeroes of the polynomial of degree $N+1: t \rightarrow P_{N+1}(t)=t \mathfrak{q}_{N}(t)-$ $r_{N} \mathfrak{q}_{N}(t)-q_{N} \mathfrak{q}_{N-1}(t)$, namely,

$$
S:=\left\{t_{k}: P_{N+1}\left(t_{k}\right)=0\right\},
$$

with $1=t_{0}>t_{1}>\cdots>t_{N} \geq-1$. The set $S$ constitutes the spectrum of $P$ (see [8, page 78]). The quantity $1-t_{1}$ is the spectral gap.

Let $\mu(d t):=\sum_{k=0}^{N} \mu_{k} \delta_{t_{k}}$ be the spectral probability measure on $[-1,1]$ with respect to which $\left(\mathfrak{q}_{y}(t), y \geq 1\right)$ are orthogonal, namely,

$$
\gamma_{y} \int_{-1}^{1} \mathfrak{q}_{x}(t) \mathfrak{q}_{y}(t) \mu(d t)=\gamma_{y} \sum_{k=0}^{N} \mu_{k} \mathfrak{q}_{x}\left(t_{k}\right) \mathfrak{q}_{y}\left(t_{k}\right)=\delta_{x, y}
$$

where $\gamma_{y}:=\pi_{y} / \pi_{0}=1 / \int_{-1}^{1} \mathfrak{q}_{y}(t)^{2} \mu(d t)=\prod_{z=0}^{y-1}\left(p_{z} / q_{z+1}\right)$, $y \geq 0$ are the potential coefficients. 
These polynomials are important in view of the KMG spectral representation theorem [8]. Indeed, with $\mathbf{q}(t)=$ $\left(\mathfrak{q}_{0}(t), \mathfrak{q}_{1}(t), \ldots, \mathfrak{q}_{N}(t)\right)^{\prime}$, we have

$$
t \mathbf{q}(t)=P \mathbf{q}(t)
$$

and therefore, $t^{n} \mathbf{q}(t)=P^{n} \mathbf{q}(t)$. Selecting the row $x$, multiplying by $\mathfrak{q}_{y}(t)$, integrating with respect to $\mu$, and applying the orthogonality relations (5), we get

$$
\begin{aligned}
P^{n}(x, y) & :=\mathbf{P}_{x}\left(X_{n}=y\right) \\
& =\gamma_{y} \int_{-1}^{1} t^{n} \mathfrak{q}_{x}(t) \mathfrak{q}_{y}(t) \mu(d t) \\
& =\gamma_{y} \sum_{k=0}^{N} \mu_{k} t_{k}^{n} \mathfrak{q}_{x}\left(t_{k}\right) \mathfrak{q}_{y}\left(t_{k}\right) .
\end{aligned}
$$

Necessarily, $\mu_{0}=\pi_{0}$ because by the ergodic theorem, for all $x$, $P^{n}(x, y) \rightarrow \mu_{0} \gamma_{y}=\left(\mu_{0} / \pi_{0}\right) \pi_{y}=\pi_{y}$ as $n \uparrow \infty$. From (7), because $\mathfrak{q}_{0}(t)=1$, we have $\mathbf{P}_{0}\left(X_{n}=0\right)=\sum_{k=0}^{N} \mu_{k} t_{k}^{n}$, and so the generating function of $\mathbf{P}_{0}\left(X_{n}=0\right)$ is

$$
\begin{aligned}
u_{0}(z) & :=\sum_{n \geq 1} z^{n} \mathbf{P}_{0}\left(X_{n}=0\right) \\
& =\sum_{k=0}^{N} \mu_{k}\left(1-z t_{k}\right)^{-1} \\
& =\int_{-1}^{1} \frac{\mu(d t)}{1-z t},
\end{aligned}
$$

the Stieltjes transform of the spectral measure, satisfying $u_{0}(1)=\infty$ as a result of $t_{0}=1$. On the other side, let $\phi_{0}(z)=\sum_{k \geq 1} z^{k} \mathbf{P}\left(\tau_{0,0}^{*}=k\right)$ be the probability generating function (pgf) of the first return time to zero $\tau_{0,0}^{*}$ probability. As it can easily be checked by renewal arguments (see [9, page 330$]), u_{0}(z)=1+u_{0}(z) \phi_{0}(z)$, showing that

$$
\begin{gathered}
u_{0}(z)=\frac{1}{1-\phi_{0}(z)}, \\
\phi_{0}(z)=1-\frac{1}{u_{0}(z)}=1-\frac{1}{\sum_{k=0}^{N} \mu_{k}\left(1-z t_{k}\right)^{-1}} .
\end{gathered}
$$

We have $\mathbf{P}\left(\tau_{0,0}^{*}<\infty\right)=\phi_{0}(1)=1$ and $\phi_{0}^{\prime}(1)=\mathbf{E}\left(\tau_{0,0}^{*}\right)=$ $\mu_{0}^{-1}=\pi_{0}^{-1}$.

More generally, with $|z| \leq 1$, let

$$
\mathfrak{g}_{z}(x, y)=\sum_{n \geq 0} z^{n} P^{n}(x, y)=(I-z P)^{-1}(x, y)
$$

be the Green potential function of the chain. We let $\mathfrak{g}(x, y):=$ $\mathfrak{g}_{1}(x, y)$, the expected sojourn time at $y$, starting from $x$. Note that $\mathfrak{g}_{z}(0,0)=u_{0}(z)$ and $\mathfrak{g}_{0}(x, y)=1$.

(i) With $\tau_{x, y}=\inf \left(n \geq 0: X_{n}=y \mid X_{0}=x\right)$ the first hitting time of $y$ starting from $x$, by renewal arguments, the pgf of $\tau_{x, y}$ reads

$$
\mathbf{E}\left(z^{\tau_{x, y}}\right)=\frac{\mathfrak{g}_{z}(x, y)}{\mathfrak{g}_{z}(x, x)},
$$

with $\tau_{x, x}=0$. We have

$$
\begin{aligned}
\mathbf{E}\left(z^{\tau_{y, x}}\right) & =\frac{\mathfrak{g}_{z}(y, x)}{\mathfrak{g}_{z}(y, y)}=\frac{\gamma_{x} \mathfrak{g}_{z}(x, x)}{\gamma_{y} \mathfrak{g}_{z}(y, y)} \frac{\mathfrak{g}_{z}(x, y)}{\mathfrak{g}_{z}(x, x)} \\
& =\frac{\gamma_{x} \mathfrak{g}_{z}(x, x)}{\gamma_{y} \mathfrak{g}_{z}(y, y)} \mathbf{E}\left(z^{\tau_{x, y}}\right) .
\end{aligned}
$$

With $x<y$, we have

$$
\begin{aligned}
& \mathbf{P}\left(\tau_{x, y}>n\right)=\mathbf{P}\left(X_{n}<y \mid X_{0}=x\right) \\
&=\sum_{0 \leq y^{\prime}<y} \mathbf{P}\left(X_{n}=y^{\prime} \mid X_{0}=x\right) \\
&=\sum_{0 \leq y^{\prime}<y} P^{n}\left(x, y^{\prime}\right), \\
& \mathbf{P}\left(\tau_{y, x}>n\right)=\mathbf{P}\left(X_{n}>x \mid X_{0}=y\right) \\
&=\sum_{N \geq y^{\prime}>x} \mathbf{P}\left(X_{n}=y^{\prime} \mid X_{0}=y\right) \\
&=\sum_{N \geq y^{\prime}>x} P^{n}\left(y, y^{\prime}\right), \\
& \mathbf{E}\left(\tau_{x, y}\right)= \sum_{n \geq 0} \mathbf{P}\left(\tau_{x, y}>n\right)=\sum_{0 \leq y^{\prime}<y} \mathfrak{g}\left(x, y^{\prime}\right), \\
& \mathbf{E}\left(\tau_{y, x}\right)=\sum_{n \geq 0} \mathbf{P}\left(\tau_{y, x}>n\right)=\sum_{N \geq y^{\prime}>x} \mathfrak{g}\left(y, y^{\prime}\right) .
\end{aligned}
$$

(ii) With $\tau_{x, x}^{*}=\inf \left(n \geq 1: X_{n}=x \mid X_{0}=x\right)$ the first return time to $x$, we have

$$
\begin{aligned}
\phi_{x}(z) & :=\mathbf{E}\left(z^{\tau_{x, x}^{*}}\right)=\frac{\mathfrak{g}_{z}(x, x)-1}{\mathfrak{g}_{z}(x, x)} \\
& =1-\frac{1}{\mathfrak{g}_{z}(x, x)}=: 1-\frac{1}{u_{x}(z)},
\end{aligned}
$$

with

$$
\mathbf{P}\left(\tau_{x, x}^{*}<\infty\right)=\phi_{x}(1)=\frac{\mathfrak{g}(x, x)-1}{\mathfrak{g}(x, x)} .
$$

The probability that the first return time to $x$ is finite is 1 if and only if $\mathfrak{g}(x, x)=\infty$ (a recurrence condition), and this happens if and only if $P$ (stochastic) is irreducible, in which case all states are in the same recurrence class. Else, if for some $x, \mathfrak{g}(x, x)<\infty$ (a transience condition for $x$ ), this probability is smaller than 1 , and one possible reason is that absorption occurs in at least one state $\neq x$; all states then but the absorbing ones (typically here, either $\{0\}$ or $\{N\}$, or both endpoints) are in the same transience class, and $\mathbf{P}\left(\tau_{x, x}^{*}<\infty\right) \in(0,1)$ because the walker may or not return to $x$ before hitting one of the absorbing states. So $P$ here is no longer irreducible, and this is a particular reducibility case where transience pops in. In the latter transient case 
however, by removing the rows and columns associated to the absorbing states, we are left with an irreducible substochastic transition matrix of smaller dimension to which the KarlinMcGregor spectral representation applies as well (see [8]). The dominant eigenvalue of the restricted matrix is now strictly smaller than 1 .

In view of $\gamma_{x}:=\pi_{x} / \pi_{0}=1 / \int_{-1}^{1} \mathfrak{q}(t)^{2} \mu(d t)$ and in case of recurrence,

$$
\mathbf{E}\left(\tau_{x, x}^{*}\right)=\frac{1}{\pi_{x}} .
$$

Equations (6) and (7) suggest that, with

$$
r_{k}(x)=\frac{\mu_{k}}{\mu_{0}} \mathfrak{q}_{x}\left(t_{k}\right), \quad l_{k}(y)=\pi_{y} \mathfrak{q}_{y}\left(t_{k}\right)
$$

the vectors $\mathbf{r}_{k}=\left(r_{k}(0), \ldots, r_{k}(x), \ldots, r_{k}(N)\right)^{\prime}$ and $\mathbf{l}_{k}^{\prime}=$ $\left(l_{k}(0), \ldots, l_{k}(y), \ldots, l_{k}(N)\right)$ can be chosen to be the right (column) and left (row) eigenvectors of $P$ associated to the eigenvalue $t_{k}$ for which:

$$
P \mathbf{r}_{k}=t_{k} \mathbf{r}_{k}, \quad \mathbf{l}_{k}^{\prime} P=t_{k} \mathbf{l}_{k}^{\prime} .
$$

In particular, $l_{0}(y)=\pi_{y} \mathfrak{q}_{y}(1)=\pi_{y}$ and $r_{0}(x)=1$, as required. We have

$$
\mathbf{l}_{j}^{\prime} \mathbf{r}_{k}=\delta_{j, k}
$$

because $\mathbf{l}_{j}^{\prime} P \mathbf{r}_{k}=t_{k} \mathbf{l}_{j}^{\prime} \mathbf{r}_{k}=t_{j} \mathbf{l}_{j}^{\prime} \mathbf{r}_{k}$, with the right normalization. Equation (19) yields another orthogonality relation which is dual to the one in (5), namely,

$$
\frac{\mu_{k}}{\mu_{0}} \sum_{x=0}^{N} \pi_{x} \mathfrak{q}_{x}\left(t_{j}\right) \mathfrak{q}_{x}\left(t_{k}\right)=\delta_{j, k} .
$$

With $P_{k}:=\mathbf{r}_{k} \mathbf{l}_{k}^{\prime}$, we now get the decomposition of $P$ into orthogonal projectors which is (7):

$$
P=\sum_{k=0}^{N} t_{k} P_{k}, \quad P^{n}=\sum_{k=0}^{N} t_{k}^{n} P_{k} .
$$

Note the biorthogonality relations:

$$
\mathbf{l}_{j}^{\prime} P \mathbf{r}_{k}=t_{k} \delta_{j, k}
$$

from which it follows that

$$
\begin{aligned}
& \sum_{x, y=0}^{N} l_{j}(x) P^{n}(x, y) r_{k}(y) \\
& \quad=\sum_{x, y=0}^{N} \pi_{x} \mathfrak{q}_{x}\left(t_{j}\right) P^{n}(x, y) \frac{\mu_{k}}{\mu_{0}} \mathfrak{q}_{y}\left(t_{k}\right) \\
& \quad=t_{k}^{n} \delta_{j, k} .
\end{aligned}
$$

There is a matrix version of all this. Calling $R$ (resp., $L$ ) the matrix whose $k$ th column (row) is $\mathbf{r}_{k}$ (resp., $\mathbf{l}_{k}^{\prime}$ ), (18) reads $P R=R D_{\mathbf{t}}$ and $L P=D_{\mathbf{t}} L$ where $D_{\mathbf{t}}:=\operatorname{diag}(\mathbf{t})$, $\mathbf{t}=\left(t_{k}, k=0, \ldots, N\right)$. Since by (19), $L R=I:=\operatorname{diag}(\mathbf{1})$, we have $L P R=D_{t} L R=D_{t}$, which is (22). Since $R L=I$, $(L P R)^{n}=D_{\mathbf{t}}^{n}=L P^{n} R$. If we let $Q$ be the matrix whose entry $(x, k)$ is $Q(x, k)=\mathfrak{q}_{x}\left(t_{k}\right)$, then $R=\left(1 / \mu_{0}\right) Q D_{\mu}$ and $L=Q^{\prime} D_{\pi}$ is the matrix formulation of (17). Let us finally check that $L$ and $R$ (i.e., (17)) are indeed admissible left and right eigen matrices. The relation $P R=R D_{\mathbf{t}}$ is also $P Q D_{\mu}=$ $Q D_{\mu} D_{\mathbf{t}}=Q D_{\mathbf{t}} D_{\mu}$ which is $P Q=Q D_{\mathbf{t}}$ which holds from (6). The relation $L P=D_{\mathbf{t}} L$ is also $Q^{\prime} D_{\pi} P=D_{\mathbf{t}} Q^{\prime} D_{\pi}$ or $\mathrm{Q}^{\prime} \overleftarrow{P^{\prime}}=D_{\mathbf{t}} \mathrm{Q}^{\prime}$ which is $\overleftarrow{P} Q=Q D_{\mathbf{t}}$ or again $P Q=Q D_{\mathbf{t}}$ because by the reversibility property, $\overleftarrow{P}=P$. If $\mu_{0} R^{2}=I(R$ is involutive), then $\mu_{0} R=L$ which means $Q^{\prime}=Q D_{\mu} D_{\pi}^{-1}$. If in addition $\boldsymbol{\mu}=\pi$, then $Q^{\prime}=Q$ : the duality relations (5) and (20) coincide, and this is an exceptional case of self duality.

The KMG spectral theory therefore gives a representation of the right and left eigenvectors of $P$ associated to its spectrum $\mathbf{t}$, in terms of the orthogonal polynomials $\mathbf{q}(t)$ evaluated at $\mathbf{t}$ (the $Q$-matrix).

The interpretation of the right eigenvectors of $P$ is as follows. We have $P^{n} \mathbf{r}_{k}=t_{k}^{n} \mathbf{r}_{k}$. Fixing the row $x$, the left-handside of the last equality is $\mathbf{E}_{x}\left[r_{k}\left(X_{n}\right)\right]$. Therefore,

$$
\mathbf{E}_{x}\left[r_{k}\left(X_{n}\right)\right]=t_{k}^{n} r_{k}(x) .
$$

Suppose that we want to compute $\mathbf{E}_{x}\left[\phi\left(X_{n}\right)\right]$ for some test function $\phi$. Let then $\phi(y)=\sum_{k=0}^{N} c_{k}(\phi) r_{k}(y)$ be the decomposition of $\phi$ in the basis $\left\{r_{k}\right\}$. We have $\left\langle r_{j}, r_{k}\right\rangle_{\pi}:=$ $\sum_{y=0}^{N} \pi_{y} r_{j}(y) r_{k}(y)=\left(\mu_{j} / \mu_{0}\right) \mathbf{l}_{j}^{\prime} \mathbf{r}_{k}=\left(\mu_{j} / \mu_{0}\right) \delta_{j, k}$.

Thus, $\left\langle r_{j}, \phi\right\rangle_{\pi}=\sum_{k=0}^{N} c_{k}(\phi)\left\langle r_{j}, r_{k}\right\rangle_{\pi}=\left(\mu_{j} / \mu_{0}\right) c_{j}(\phi)$ and

$$
\begin{aligned}
\mathbf{E}_{x}\left[\phi\left(X_{n}\right)\right] & =\sum_{k=0}^{N} t_{k}^{n} \cdot \frac{\mu_{0}}{\mu_{k}}\left\langle r_{k}, \phi\right\rangle_{\pi} \cdot r_{k}(x) \\
& =\frac{1}{\pi_{x}} \sum_{k=0}^{N} t_{k}^{n} \cdot\left\langle r_{k}, \phi\right\rangle_{\pi} \cdot l_{k}(x) .
\end{aligned}
$$

Whenever the spectral structure of the chain is known, the solution $\mathbf{E}_{x}\left[\phi\left(X_{n}\right)\right]$ to the backward Kolmogorov equations are thus computable exactly. Let us now investigate some special classes of random walks (RWs).

2.3. Symmetric RW. Suppose that $N$ is even with $N=2 N_{0}$. Assume that a random walk $X_{n}$ on $\left\{0, \ldots, 2 N_{0}\right\}$ is given so that

$$
P=\left[\begin{array}{ccccc}
0 & 1 & & & \\
q_{1} & 0 & p_{1} & & \\
& \ddots & \ddots & \ddots & \\
& & q_{2 N_{0}-1} & 0 & p_{2 N_{0}-1} \\
& & & 1 & 0
\end{array}\right]
$$

is the transition matrix of some symmetric random walk reflected at the boundaries $\left\{0,2 N_{0}\right\}$. The KMG theory is also relevant for such transition matrices $P^{n}$. Let $t_{k}, k=$ $0, \ldots, 2 N_{0}$ be the zeroes of the polynomial of degree $2 N_{0}+1$ : $t \rightarrow P_{2 N_{0}+1}(t)=t \mathfrak{q}_{2 N_{0}}(t)-\mathfrak{q}_{2 N_{0}} \mathfrak{q}_{2 N_{0}-1}(t)$, namely,

$$
S=\left\{t_{k}: P_{2 N_{0}+1}\left(t_{k}\right)=0\right\} \text {, }
$$


with $1=t_{0}>t_{1} \geq \cdots \geq t_{2 N_{0}}=-1$. Then, $\mu(d t):=\sum_{k=0}^{2 N_{0}} \mu_{k} \delta_{t_{k}}$ is the probability measure on $[-1,1]$ with respect to which the polynomial $\left(\mathfrak{q}_{y}(t), y \geq 1\right)$ associated to $P$ is orthogonal. This measure is symmetric on $[-1,1]$. In particular, $t_{N_{0}}=0$ and $t_{2 N_{0}}=-1, t_{0}=1$ are eigenvalues of such Ps. When $N$ is odd, say with $N=2 N_{0}+1$, the spectral measure $\mu(d t):=\sum_{k=0}^{2 N_{0}+1} \mu_{k} \delta_{t_{k}}$ is again symmetric, but $\{0\}$ no longer is an eigenvalue, and $t_{N_{0}}>0$.

Example 1. The simplest such example is the simple gambler random walk $X_{n}$ for which $p_{x}=p, q_{x}=q, x=1, \ldots, N-$ $1(p+q=1)$ with a pure reflection at the endpoints $\left(p_{0}=q_{N}=1\right)$. The invariant measure $\pi$ is a truncated geometric distribution. It follows from [9, page 438] that $t_{k}=$ $2 \sqrt{p q} \cos (k \pi / N), k=1, \ldots, N-1, t_{0}=1, t_{N}=-1$. The spectral gap tends to $1-2 \sqrt{p q} \neq 0$ as $N \uparrow \infty$ if $p \neq 1 / 2$. The mass of the spectral measure can be seen to be equal to $\mu_{N}=\mu_{0}=\pi_{0}=(1-(p / q)) /\left[2\left(1-(p / q)^{N}\right)\right], \mu_{k}=$ $\left(1-2 \mu_{0}\right) /(N-1), k=1, \ldots, N-1$ (with $\mu_{0}=1 /(2 N)$ when $p=$ $1 / 2)$. It exhibits boundary effects causing deviation from the uniform measure on $\{0, \ldots, N\}$. The orthogonal polynomials involve two sine functions. A closed form expression of the generating functions of $\mathbf{P}_{0}\left(X_{n}=0\right)$ and $\mathbf{P}\left(\tau_{0,0}^{*}=k\right)$ follows.
Additional Comment. Let $P$ be the tridiagonal transition matrix associated to this RW $X_{n}$. Let $\bar{P}$ be the transition matrix associated to $\bar{X}_{n}:=N-X_{n}$. Then, $\bar{P}(x, y)=P(N-$ $x, N-y$ ) has the same structure as $P$ except that $p$ and $q$ are interchanged (a drift reversal property). $\bar{P}$ is obtained from $P$ by applying a permutation reversing the order of the rows and then of the columns. So $\bar{P}$ and $P$ share the same spectrum, and this is why $t_{k}$ is a symmetric function of $p$ and $q$. However, the mass of the spectral measure of $\bar{P}$ is different with: $\bar{\mu}_{N}=\bar{\mu}_{0}=(1-(q / p)) /\left[2\left(1-(q / p)^{N}\right)\right]$.

\subsection{Special Cases: Spectral Positivity. Let}

$$
S^{\prime}=\left[\begin{array}{ccccc}
\sqrt{p_{0}} & & & & \\
\sqrt{q_{1}} & \sqrt{p_{1}} & & & \\
& \ddots & \ddots & & \\
& & \sqrt{q_{N-1}} & \sqrt{p_{N-1}} & \\
& & & \sqrt{q_{N}} & \sqrt{p_{N}}
\end{array}\right]
$$

be a subdiagonal matrix. It is the transpose of a superdiagonal matrix $S$. Then, with $D_{\mathbf{r}}:=\operatorname{diag}\left(r_{0}, \ldots, r_{N}\right)$

$$
\begin{aligned}
& S^{\prime} S=\left[\begin{array}{ccccc}
p_{0} & \sqrt{p_{0} q_{1}} & & & \\
\sqrt{p_{0} q_{1}} & p_{1}+q_{1} & \sqrt{p_{1} q_{2}} & & \\
& \ddots & \ddots & \ddots & \\
& & \sqrt{p_{N-2} q_{N-1}} & p_{N-1}+q_{N-1} & \sqrt{p_{N-1} q_{N}} \\
& & & \sqrt{p_{N-1} q_{N}} & p_{N}+q_{N}
\end{array}\right] \\
& =I-D_{\mathbf{r}}+\left[\begin{array}{ccccc}
0 & \sqrt{p_{0} q_{1}} & & & \\
\sqrt{p_{0} q_{1}} & 0 & \sqrt{p_{1} q_{2}} & & \\
& \ddots & \ddots & \ddots & \\
& & \sqrt{p_{N-2} q_{N-1}} & 0 & \sqrt{p_{N-1} q_{N}} \\
& & & \sqrt{p_{N-1} q_{N}} & 0
\end{array}\right] \text {. }
\end{aligned}
$$

Thus,

$$
P_{S}=2 D_{\mathbf{r}}-I+S^{\prime} S
$$

is the sum of a diagonal matrix and a symmetric positive definite matrix. From this, we conclude that if the holding probabilities $r_{x} \geq 1 / 2$, for all $x=0, \ldots, N$, then for all $\mathbf{z} \in \mathbb{R}^{N+1} \backslash\{\mathbf{0}\}$,

$$
\mathbf{z}^{\prime} P_{S} \mathbf{z}=\sum_{x=0}^{N}\left(2 r_{x}-1\right)\left|z_{x}\right|^{2}+|S \mathbf{z}|^{2}>0,
$$

and so $P_{S}$ and then $P$ are positive definite with real positive eigenvalues. The Jacobi matrix $P$ then has all its principal minors nonnegative and therefore is oscillatory (i.e., totally nonnegative and such that $P^{N}$ is totally positive) with all its minors nonnegative (see e.g., [10, page 99]). Oscillatory stochastic matrices have distinct positive eigenvalues, with: $1=t_{0}>t_{1}>\cdots>t_{N}>0$. As a sequence of numbers, the right row eigenvector $\mathbf{r}_{k}=\left(r_{k}(0), \ldots, r_{k}(x), \ldots, r_{k}(N)\right)^{\prime}$ associated to $t_{k}$ has exactly $k$ sign changes (see [10, page 101]).
And so do the numbers: $\left(\mathfrak{q}_{x}\left(t_{k}\right) ; x=0, \ldots, N\right)$. The above condition $r_{x} \geq 1 / 2$ is a simple sufficient condition allowing to exhibit examples of $P$ that are oscillatory but of course, it is not necessary; the full condition under our assumptions being $\mathbf{z}^{\prime} P_{S} \mathbf{z}>0$ for all $\mathbf{z} \in \mathbb{R}^{N+1} \backslash\{\mathbf{0}\}$ or

$$
\mathbf{z}^{\prime} P \mathbf{z}=\sum_{x=0}^{N} r_{x} z_{x}^{2}+\sum_{x=0}^{N-1}\left(p_{x}+q_{x+1}\right) z_{x} z_{x+1}>0
$$

Equivalently, if $s_{k}$ are the positive eigenvalues of $S^{\prime} S$, we need to check $2 r_{k}-1+s_{k}>0$ for all $k$.

For a simple random walk $X_{n}$ whose $(N+1)^{2}$-transition matrix $P$ is spectrally nonnegative (resp., spectrally positive), there exists a symmetric random walk $Y_{m}$ on $\{0, \ldots, 2 N\}$ (resp., on $\{0, \ldots, 2 N+1\}$ ), reflected at the endpoints, started at an even integer $\{0,2,4, \ldots, 2 N\}$ (resp., odd integer $\{1,3, \ldots, 2 N+1\}$ ), such that $\left\{X_{n}\right\} \stackrel{d}{=}\left\{Y_{2 n} / 2\right\}$, (resp., $\left\{X_{n}\right\} \stackrel{d}{=}$ $\left.\left\{\left(Y_{2 n}-1\right) / 2\right\}\right)$. This follows simply from adapting [11, Theorem 2.1] to our finite-dimensional case. As noted just before, the spectral measure of the random walk $Y_{m}$ is symmetric on 
$[-1,1]$, and by passing to $X_{n}$, the spectrum is being folded: If $\sum_{k=0}^{2 N} \mu_{k} \delta_{t_{k}}$ (resp., $\sum_{k=0}^{2 N+1} \mu_{k} \delta_{t_{k}}$ ) is the symmetric spectral measure of $Y_{m}$ with $t_{N}=0$ (resp., $t_{N}>0$ ), then $2 \sum_{k=0}^{N} \mu_{k} \delta_{t_{k}^{2}}$ is the spectral measure of $X_{n}$. Let $\alpha_{y}$ and $\beta_{y}$ be the up and down probabilities that $Y_{m} \rightarrow Y_{m+1}=Y_{m} \pm 1$ in one step given $Y_{m}$ is in state $y$ different from the endpoints, $\alpha_{y}+\beta_{y}=1$, then

$$
\begin{gathered}
q_{x}=\beta_{2 x} \beta_{2 x-1}, \quad r_{x}=\beta_{2 x} \alpha_{2 x-1}+\alpha_{2 x} \beta_{2 x+1}, \\
p_{x}=\alpha_{2 x} \alpha_{2 x+1} .
\end{gathered}
$$

This, together with $p_{0}=\alpha_{1}$ and $q_{N}=\beta_{2 N-1}\left(\right.$ resp., $\left.q_{N}=\beta_{2 N}\right)$, allows to determine recursively the transition matrix of $Y_{m}$ from the one of $X_{n}$. From these facts, we get the following proposition.

Proposition 2. If a $B D$ chain $X_{n}$ is spectrally nonnegative, then it is stochastically monotone.

Proof. Under our hypothesis indeed,

$$
p_{x}+q_{x+1}=\alpha_{2 x} \alpha_{2 x+1}+\beta_{2 x+2} \beta_{2 x+1}<\alpha_{2 x} \alpha_{2 x+1}+\beta_{2 x+1}<1,
$$

because this is also $\alpha_{2 x}<1$. This condition means that $X_{n}$ is stochastically monotone (see below the construction of the Siegmund dual of an RW where stochastic monotonicity is needed). See also [6, Lemma 2.4] for a different proof.

Remark 3. Suppose a $\mathrm{BD}$ chain $X_{n}$ with transition matrix $P$ is not spectrally nonnegative, so $P$ has strictly negative eigenvalues $\left(t_{N}<0\right)$. With $\rho \in(0,1)$, consider the lazy modified stochastic matrix

$$
P_{\rho}=(1-\rho) I+\rho P \text {. }
$$

The eigenvalues of $P_{\rho}$ are $t_{k}^{(\rho)}=1-\rho+\rho t_{k}, k=0, \ldots, N$ with the same left and right eigenvectors as the ones of $P$. One can always choose $\rho \leq\left(1-t_{N}\right)^{-1}$ small enough so that $t_{N}^{(\rho)} \geq 0$. For this $\rho, P_{\rho}$ is now spectrally nonnegative, and so $P_{\rho}$ governs a stochastically monotone chain $X_{n}^{(\rho)}$. The RW $X_{n}^{(\rho)}$, with shifted spectrum, clearly has the same invariant measure as the one of $X_{n}$.

For example, solving (34) with the boundary conditions $p_{0}=\alpha_{1}$ and $q_{N}=\beta_{2 N}$ gives the forward and backward recurrences of the unknown odd and even probability terms in the bulk:

$$
\begin{aligned}
& \text { For } x=1, \ldots, N-1: \\
& \qquad \begin{array}{r}
\alpha_{2 x+1}=\frac{p_{x}}{\left(1-q_{x} /\left(1-\alpha_{2 x-1}\right)\right)}, \\
\alpha_{1}=p_{0},
\end{array}
\end{aligned}
$$

For $x=N-1, \ldots, 1$ :

$$
\begin{gathered}
\alpha_{2 x}=\frac{p_{x}}{\left(1-q_{x+1} /\left(1-\alpha_{2(x+1)}\right)\right)}, \\
\alpha_{2 N}=1-q_{N} .
\end{gathered}
$$

As a conclusion, it is easy to construct spectrally positive RWs by "squaring" two symmetric random walks, but given a nonsymmetric RW, it may be difficult to decide whether it is or not spectrally positive because one needs conversely to check whether all the above $\alpha$ s are probabilities. Spectral positivity determines stochastic monotonicity.

2.5. Scale Function and Excursion Height of an RW. Assume the height $H=h \in\{1, \ldots, N-1\}$ for some excursion (sample paths of $X_{n}$ between two consecutive visits to state 0 ). This event will be realized if and only if (i) downward paths started from $h$ hit state $\{0\}$ before hitting state $h+1$ and (ii) upward paths started at $\{0\}$ first reach 1 (with probability $p_{0}$ ) and then, paths started at 1 hit $h$ without returning to $\{0\}$ again in the intervening time. These two events are independent. Therefore, with $\tau_{x, y}$, the first hitting time of $y$ starting from $x$ :

$$
\begin{array}{r}
\mathbf{P}(H=h)=p_{0} \mathbf{P}\left(\tau_{1, h}<\tau_{1,0}\right) \mathbf{P}\left(\tau_{h, 0}<\tau_{h, h+1}\right), \\
h=1, \ldots, N .
\end{array}
$$

Assume $X_{0}=x$. Let $X_{n \wedge \tau_{x, 0}}$ denote the random walk stopped when it first hits 0 . Its transition matrix is $P$ except for its first row which is $(1,0,0, \ldots, 0)$, translating that state $\{0\}$ is now absorbing. Define the scale (or harmonic) function $\varphi$ of this random walk as the function which makes $M_{n}:=\varphi\left(X_{n \wedge \tau_{x, 0}}\right)$ a martingale. The function $\varphi$ is important because, as is wellknown, for all $0<x<h \leq N$, with $\tau_{x}=\tau_{x, 0} \wedge \tau_{x, h}$, the first hitting time of $\{0, h\}$ starting from $x$

$$
\mathbf{P}\left(X_{\tau_{x}}=h\right)=\frac{\varphi(x)}{\varphi(h)} .
$$

Using this remark, we get

$$
\begin{gathered}
\mathbf{P}(H=h)=p_{0} \mathbf{P}\left(\tau_{1, h}<\tau_{1,0}\right) \mathbf{P}\left(\tau_{h, 0}<\tau_{h, h+1}\right) \\
=p_{0} \frac{\varphi(1)}{\varphi(h)}\left(1-\frac{\varphi(h)}{\varphi(h+1)}\right), \\
h \in\{1, \ldots, N-1\} .
\end{gathered}
$$

Note that $\mathbf{P}(H \geq h)=p_{0} / \varphi(h)$ if $\varphi(1)=1$. The event $H=N$ may also occur, with probability $\mathbf{P}(H=N)=p_{0} / \varphi(N)$.

The interpretation the event $H=N$ takes on is twofold: either it is the maximal height of an excursion if the state $\{N\}$ remains reflecting, either, if state $\{N\}$ is itself assumed absorbing, $\mathbf{P}\left(X_{\tau_{x}}=N\right)=\varphi(x) / \varphi(N)=\left(\varphi(x) / p_{0}\right) \mathbf{P}(H=N)$ constitutes the probability of a fixation event at $\{N\}$, starting from state $x$. We can thus compute the probability of a fixation before extinction at $\{0\}$ occurs. It remains to compute $\varphi$ (defined up to an arbitrary multiplicative constant). We wish to have $\mathbf{E}_{x}\left(M_{n+1} \mid M_{n}=y\right)=y$, leading to $\varphi(0)=0$ and

$$
\varphi(x)=q_{x} \varphi(x-1)+r_{x} \varphi(x)+p_{x} \varphi(x+1), \quad x \geq 1,
$$

where $p_{x}+q_{x}+r_{x}=1, x \geq 1$. Imposing $\varphi(1)=1$ (fixing the arbitrary multiplicative constant), the searched "harmonic" 
function is $\varphi(x)=: 1+\sum_{y=1}^{x-1} \psi(y)$ where $\psi(y)$ satisfies $q_{y} \psi(y-$ $1)=p_{y} \psi(y)$. Thus, $\psi(y)=\prod_{z=1}^{y}\left(q_{z} / p_{z}\right)$ and

$$
\varphi(x)=1+\sum_{y=1}^{x-1} \prod_{z=1}^{y} \frac{q_{z}}{p_{z}}, \quad x \geq 1, \varphi(0):=0 .
$$

Equations (39) and (41) characterize the law of the excursion height of the random walk $X_{n}$. We note that $\varphi(x)$ increases with $x$. From (38) and (41), we get an explicit expression of the probability of the event $\tau_{x, h}<\tau_{x, 0}$ :

$$
\mathbf{P}\left(\tau_{x, h}<\tau_{x, 0}\right)=\mathbf{P}\left(X_{\tau_{x}}=h\right)=\frac{\varphi(x)}{\varphi(h)},
$$

where $\tau_{x, h}$ is the first hitting time of $h$ starting from $x$, with $0<$ $x<h \leq N$. In particular, if $h=N$ with $\{N\}$ also absorbing, $\mathbf{P}\left(\tau_{x, N}<\tau_{x, 0}\right)=\varphi(x) / \varphi(N)$ is the fixation probability starting from $x$, whereas $\mathbf{P}\left(\tau_{x, 0}<\tau_{x, N}\right)=1-\mathbf{P}\left(\tau_{x, N}<\tau_{x, 0}\right)$ is the extinction probability.

\section{A BD Example: The Moran Model}

The example we have in mind is the 2-allele Moran model with bias mechanism $p$. Let

$$
p(u): u \in[0,1] \longrightarrow[0,1], \quad \text { with } 0 \leq p(0), p(1) \leq 1
$$

be continuous and $\bar{p}(u):=1-p(u)$.

The Moran model $X_{n}$ is characterized by the following transition probabilities, given the walker is in state $x \in$ $\{0, \ldots, N\}$ :

$$
\begin{gathered}
q_{x}=\frac{x}{N} \bar{p}\left(\frac{x}{N}\right), \\
r_{x}=\frac{x}{N} p\left(\frac{x}{N}\right)+\left(1-\frac{x}{N}\right) \bar{p}\left(\frac{x}{N}\right), \\
p_{x}=\left(1-\frac{x}{N}\right) p\left(\frac{x}{N}\right) .
\end{gathered}
$$

For a Moran model $X_{n}$ is the amount of say allele or type 1 individuals in a population of size $N$, and the reproduction law is obtained while choosing two individuals at random among $N$, one dying and the other one passing to the next generation while giving birth to an additional individual so as to keep constant the full population size $N$ over the generations. Given then $X_{n}=x$, the transition $X_{n} \rightarrow X_{n}+1$ $\left(X_{n} \rightarrow X_{n}-1\right)$ occurs with probability $p_{x}\left(q_{x}\right)$ translating the fact that the individual bound to die is chosen among type 2 (type 1 ) individuals with probability $1-x / N(x / N)$, and the one which splits into two new born is chosen among the other type, with probability $p(x / N)$ (resp., $\bar{p}(x / N))$, after deforming the neutral frequency $x / N$ by the bias mechanism $p$. These bias $p$ take into account various evolutionary forces which are the causes of deviation to neutrality (see [12-14] for a discussion on various models of utmost interest in population genetics, among which selection and mutations). The classical neutral Moran model is obtained when $p(u)=u$ (no external deformation). Extended Moran models are studied in [15].

Assuming $p_{0}=p(0)>0$ and $q_{N}=1-p(1)>0$, with $y \in\{1, \ldots, N\}$, the corresponding Markov chain is ergodic with invariant distribution:

$$
\frac{\pi_{y}}{\pi_{0}}=\prod_{z=0}^{y-1} \frac{p_{z}}{q_{z+1}}=\left(\begin{array}{c}
N \\
y
\end{array}\right) \prod_{z=0}^{y-1} \frac{p(z / N)}{\bar{p}((z+1) / N)},
$$

where

$$
\pi_{0}=\frac{1}{1+\sum_{y=1}^{N}\left(\begin{array}{c}
N \\
y
\end{array}\right) \prod_{z=0}^{y-1}(p(z / N) / \bar{p}((z+1) / N))} .
$$

We have the following obvious facts.

Proposition 4. Let $X_{n}$ be a Moran process with transition probabilities (44) for some bias $p$. Then, $\bar{X}_{n}:=N-X_{n}$ is again a Moran process with transition probabilities of the type (44) but with the new bias $\widetilde{p}(u):=1-p(1-u)$, namely, $\bar{q}_{x}=$ $p_{N-x}=(x / N)(1-\widetilde{p}(x / N)), \bar{p}_{x}=q_{N-x}=(1-x / N) \widetilde{p}(x / N)$. The spectrum of the transition matrix $\bar{P}$ of $\bar{X}_{n}$ is the same as the one $P$ of $X_{n}$. If the bias is such that $\widetilde{p}(u)=p(u)$, then the transition matrix of $\bar{X}_{n}$ coincides with the one of $X_{n}$.

Proof. We have $\bar{P}(x, y)=P(N-x, N-y)$, and $\bar{P}$ is obtained from $P$ applying the permutation $\sigma:(0,1, \ldots, N) \rightarrow$ $(N, \ldots, 1,0)$ reversing the order of the rows and then of the columns.

3.1. Fixation Probability. Forcing the states $\{0, N\}$ to be absorbing, the scale function reads

$$
\begin{gathered}
\varphi(0)=0, \\
\varphi(x)=1+\sum_{y=1}^{x-1} \frac{1}{\left(\begin{array}{c}
N-1 \\
y
\end{array}\right)} \prod_{z=1}^{y} \frac{\bar{p}(z / N)}{p(z / N)},
\end{gathered}
$$

with $\varphi(x) / \varphi(N)$ being the fixation probability, whereas 1 $\varphi(x) / \varphi(N)$ is the extinction probability, starting from $x$ (for the neutral model with $p(u)=u$, the scale function is $\varphi(x)=x$, and the fixation probability is trivially $x / N)$. The probability of a fixation before extinction occurs in an excursion reads

$$
\begin{aligned}
\mathbf{P}(H & =N) \\
& =\frac{p_{0}}{\varphi(N)} \\
& =\frac{p(0)}{1+\sum_{y=1}^{N-1}\left(1 /\left(\begin{array}{c}
N-1 \\
y
\end{array}\right)\right) \prod_{z=1}^{y}(\bar{p}(z / N) / p(z / N))} .
\end{aligned}
$$

We have

$$
\mathbf{P}(H=N)=p_{0} \mathbf{P}\left(\tau_{1, N}<\tau_{1,0}\right),
$$

where $\mathbf{P}\left(\tau_{1, N}<\tau_{1,0}\right)=1 / \varphi(N)$ is the fixation probability of a mutant-type allele $A_{1}$, for example, the probability that, starting from $X_{0}=1$, the RW hits state $\{N\}$ (fixation of the mutant-type allele 1 ) before hitting state $\{0\}$ (extinction of allele 1). 
3.2. Examples of Bias Mechanisms. We now give three examples of bias that we will deal with in the sequel.

Moran Model with Mutations. A basic bias example is the mutation mechanism

$$
p(u)=\left(1-\mu_{2}\right) u+\mu_{1}(1-u)
$$

where $\left(\mu_{1}, \mu_{2}\right)$ are mutation probabilities in $(0,1]$. We let $\bar{\mu}_{1}:=1-\mu_{1}, \bar{\mu}_{2}:=1-\mu_{2}, \mu=\mu_{1}+\mu_{2}$, and $\bar{\mu}=1-\mu$.

Schematically, type 1 alleles are being produced whenever type 1 alleles do not mutate to type 2 (with probability $\bar{\mu}_{2}$ ) or type 2 alleles mutate to type 1 (with probability $\mu_{1}$ )

$$
\stackrel{\bar{\mu}_{2}}{\circlearrowleft} A_{1} \underset{\mu_{1}}{\stackrel{\mu_{2}}{\rightleftharpoons}} A_{2} \stackrel{\bar{\mu}_{1}}{\circlearrowright}
$$

The transition matrix $P$ of this model has therefore the particular $\mathrm{BD}$ structure

$$
\begin{gathered}
q_{x}=\frac{x}{N}\left(\bar{\mu}_{1}-\bar{\mu} \frac{x}{N}\right), \\
r_{x}=1-\left(p_{x}+q_{x}\right), \\
p_{x}=\left(1-\frac{x}{N}\right)\left(\mu_{1}+\bar{\mu} \frac{x}{N}\right),
\end{gathered}
$$

with $p_{0}=\mu_{1}, q_{N}=\mu_{2}$. When $\mu \neq 1$, we introduce the auxiliary parameters

$$
v_{1}=\frac{\mu_{1}}{\bar{\mu}}, \quad v_{2}=\frac{\mu_{2}}{\bar{\mu}}, \quad v=v_{1}+v_{2}=\frac{\mu}{\bar{\mu}}
$$

with $\nu_{1}+1=\bar{\mu}_{2} / \bar{\mu}$ and $\nu_{2}+1=\bar{\mu}_{1} / \bar{\mu}$.

The drift of this RW is

$$
\begin{aligned}
\mathrm{E}\left(X_{n+1}-X_{n} \mid X_{n}=x\right) & =p_{x}-q_{x} \\
& =p\left(\frac{x}{N}\right)-\frac{x}{N} \\
& =\mu_{1}-\mu \frac{x}{N} .
\end{aligned}
$$

The deterministic part of the dynamics of $X_{n}$ has a stable point at $x=N \mu_{1} / \mu$, cancelling the drift. When $p$ is nondecreasing, we have $\mu \leq 1$. We note that in the expression of $\widetilde{p}(u)$, the roles of $\mu_{1}$ and $\mu_{2}$ are interchanged. So, $\widetilde{p}(u)=$ $p(u)$ if and only if $\mu_{1}=\mu_{2}$ (symmetric mutations).

If $\mu_{1}=\mu_{2}=1$, we recover if $N$ is even the heat-exchange Bernoulli-Laplace model [9] as a borderline example, but $p(u)=1-u$ is strictly decreasing in this case. If $\mu_{1}=$ $\mu_{2}=1 / 2$, then $p(u)=1 / 2$, and we obtain an aperiodic model amenable (through a suitable time substitution) to the Ehrenfest urn model provided $N$ is even.

For one-way mutations, $\left(\mu_{1}, \mu_{2}\right)=\left(\mu_{1}, 0\right)$ or $\left(0, \mu_{2}\right)$ leading to the simpler forms $\mu_{1}+u \bar{\mu}_{1}$ and $\bar{\mu}_{2} u$ of $p(u)$, but with $p(1)=1$ and $p(0)=0$, respectively, corresponding to $\{N\}$ (resp., $\{0\}$ ) being absorbing. We avoid this situation because it deserves another treatment due to transience.

Moran Model with Mutations and Selection. Another example could be

$$
p(u)=\frac{\mu_{1}+u\left((1+s) \bar{\mu}_{2}-\mu_{1}\right)}{1+s u},
$$

which is the composition of the selection bias mechanism

$$
p(u)=\frac{(1+s) u}{1+s u} \quad(s>-1)
$$

with the mutation mechanism (50). When considering the fixation probability $\mathbf{P}(H=N)$ for the Moran model with this particular mechanism, we obtain a closed-form formula for the fixation probability of a mutant which of course is an interesting issue in genetics.

Cases with Positive Eigenvalues. We may look for conditions on the mechanism $p$ leading to $r_{x} \geq 1 / 2$ in which case the RW is spectrally positive. Assume $p(u): u \in(0,1) \rightarrow(0,1)$ is continuous and nondecreasing, with $0<p(0) \leq p(1)<1$.

Then, as can easily be checked: $r_{x} \geq 1 / 2$ for all $x$ if and only if $p(1 / 2)=1 / 2$ with $p(0) \leq 1 / 2 \leq p$ (1). Indeed, imposing $r_{x} \geq 1 / 2$ for all $x$ leads to $p(u) \geq 1 / 2$ if $u \geq 1 / 2$ and $p(u) \leq 1 / 2$ if $u \leq 1 / 2$. So, if $p$ is nondecreasing with $p(1 / 2)=1 / 2$, then $r_{x} \geq 1 / 2$.

(i) Take, for example, the mutation mechanism now satisfying $0<\mu_{1} \leq \bar{\mu}_{2}<1$. The condition $p(1 / 2)=$ $1 / 2$ leads to $\mu_{1}=\mu_{2}$ (fair mutation). In this case, $p(u)=\mu_{1}+\left(1-2 \mu_{1}\right) u$ with $0<\mu_{1} \leq 1 / 2$. If $\mu_{1}=1 / 2$, then $p(u)=1 / 2$ and $q_{x}=x / 2 N, r_{x}=1 / 2$, and $p_{x}=(1 / 2)(1-x / N)$.

For example, if $N=2$,

$$
P=\left[\begin{array}{ccc}
\bar{\mu}_{1} & \mu_{1} & 0 \\
\frac{1}{4} & \frac{1}{2} & \frac{1}{4} \\
0 & \mu_{1} & \bar{\mu}_{1}
\end{array}\right]
$$

has three distinct real nonnegative eigenvalues $\left\{1, \bar{\mu}_{1},\left(1-2 \mu_{1}\right) / 2\right\}$ ordered in decreasing size. We note however that it is not necessary that $\mu_{1}=\mu_{2}$ for $P$ to be spectrally positive; indeed, for the full Moran model with mutations $\mu_{1} \neq \mu_{2}$, under the sole condition $\mu_{1}+\mu_{2} \leq 1$ ( $p$ is nondecreasing), the eigenvalues $t_{k}$ can be seen to be nonnegative. Still, we were not able to find a sharp general condition on $p$ that would lead to a spectrally nonnegative Moran chain.

(ii) For the mechanism $p$ of (55) combining mutations with selection, assuming $0<\mu_{1} \leq \bar{\mu}_{2}<1$ to guarantee that $p$ is nondecreasing, the condition $p(1 / 2)=1 / 2$ relates $\left(s, \mu_{1}, \mu_{2}\right)$ to give $s=\left(\mu_{2}-\right.$ $\left.\mu_{1}\right) /\left(1 / 2-\mu_{2}\right)$, where both $\mu_{1}, \mu_{2}<1 / 2$. In this case, the model is spectrally positive. 
3.3. Fixation Probabilities for the Moran Model with Mutations and/or Selection. We now study the particular Moran model with mutations and/or selection, starting with fixation probabilities.

Moran with Mutations (sharp threshold phenomenon). We need to evaluate $\varphi(N)=1+\sum_{y=1}^{N-1}\left(1 /\left(\begin{array}{c}N-1 \\ y\end{array}\right)\right) \prod_{z=1}^{y}(\bar{p}(z / N) /$ $p(z / N))$ for large $N$ and then $\mathbf{P}\left(\tau_{1, N}<\tau_{1,0}\right)=1 / \varphi(N)$ will follow.

(i) Suppose first that $\mu=1$ and let $\rho:=\bar{\mu}_{1} / \mu_{1}$.

Proposition 5. The fixation probability satisfies the sharp threshold property:

(i) if $\mu_{1}=1 / 2(\rho=1)$, then $\mathbf{P}\left(\tau_{1, N}<\tau_{1,0}\right)=1 / \varphi(N) \rightarrow$ $1 / 2$;

(ii) if $\mu_{1}<1 / 2(\rho>1)$, then $\mathbf{P}\left(\tau_{1, N}<\tau_{1,0}\right)=1 / \varphi(N) \sim$ $\rho^{-N} \rightarrow 0$;

(iii) if $\mu_{1}>1 / 2(\rho<1)$, then $\mathbf{P}\left(\tau_{1, N}<\tau_{1,0}\right)=1 / \varphi(N) \rightarrow$ 1.

Proof. With $\rho=\bar{\mu}_{1} / \mu_{1}$, we first have the identity for sums of inverse binomial coefficients (see e.g., [16, page 197])

$$
\begin{aligned}
\varphi(N) & :=\sum_{y=0}^{N-1} \frac{\rho^{y}}{\left(\begin{array}{c}
N-1 \\
y
\end{array}\right)} \\
& =\frac{N}{(\rho+1)(1 / \rho+1)^{N}} \sum_{k=1}^{N} \frac{\left(\rho^{k}+1\right)(1 / \rho+1)^{k}}{k} .
\end{aligned}
$$

There is therefore a closed-form expression of the fixation probability $\mathbf{P}\left(\tau_{1, N}<\tau_{1,0}\right)=1 / \varphi(N)$. Observing now

$$
\begin{aligned}
\varphi(N) & :=\sum_{y=0}^{N-1} \frac{\rho^{y}}{\left(\begin{array}{c}
N-1 \\
y
\end{array}\right)} \\
& =1+\frac{\rho}{N-1}+\frac{\rho^{N-2}}{N-1}+\rho^{N-1}+\sum_{y=2}^{N-3} \frac{\rho^{y}}{\left(\begin{array}{c}
N-1 \\
y
\end{array}\right)},
\end{aligned}
$$

we get

(i) $\bar{\mu}_{1}=\mu_{1}(\rho=1): \varphi(N) \rightarrow 2^{+}$. So: $\mathbf{P}\left(\tau_{1, N}<\tau_{1,0}\right)=$ $1 / \varphi(N) \rightarrow 1 / 2$

(ii) $\bar{\mu}_{1}>\mu_{1}(\rho>1): \varphi(N) \sim \rho^{N-1}$. So: $\mathbf{P}\left(\tau_{1, N}<\tau_{1,0}\right)=$ $1 / \varphi(N) \sim \rho^{-N} \rightarrow 0$

(iii) $\bar{\mu}_{1}<\mu_{1}(\rho<1): \varphi(N) \rightarrow 1^{+}$. So: $\mathbf{P}\left(\tau_{1, N}<\tau_{1,0}\right)=$ $1 / \varphi(N) \rightarrow 1$.

If $\rho=1$, because $1 /\left(\begin{array}{c}N \\ y\end{array}\right) \leq 1 /\left(\begin{array}{c}N \\ 2\end{array}\right)$ if $y \in\{2, N-2\}: 2+2 / N<$ $\varphi(N+1)<2+2 / N+2(N-3) /(N(N-1))$ and $\varphi_{1}(N+1)$ is very close to $2+2 / N$. The fixation probability tends to $1 / 2$ from below.

If $\rho \neq 1,1+\rho^{N}+\rho / N+\rho^{N-1} / N<\varphi(N+1)<1+\rho^{N}+$ $\rho / N+\rho^{N-1} / N+2 /(N(N-1)) \rho^{2}\left(\left(1-\rho^{N-3}\right) /(1-\rho)\right)$, and $\varphi(N+1)$ is very close to $\rho^{N}$ if $\rho>1$ and very close to 1 up to a $\rho / N$ factor if $\rho<1$.
When $\mu_{1}<1 / 2$, the probability, starting from $\{0\}$ to hit the state $\{N\}$ (fixation) starting from 1 before returning to $\{0\}$, tends to 0 geometrically fast. On the contrary, would $\mu_{1}>1 / 2$, this probability would converge to 1 ; mutations producing type 1 individuals are sufficiently large to enhance fixation of a mutant allele with probability 1 . If $\mu_{1}=1 / 2$, the fixation probability approaches $1 / 2$.

(ii) Suppose now that $\mu \neq 1$. With $A_{N}=N\left(\bar{\mu}_{1} / \bar{\mu}\right)=$ $N\left(\nu_{2}+1\right), B_{N}=N\left(\mu_{1} / \bar{\mu}\right)=N v_{1}$,

$$
\begin{aligned}
\varphi(N) & =1+\sum_{y=1}^{N-1} \frac{1}{\left(\begin{array}{c}
N-1 \\
y
\end{array}\right)} \prod_{z=1}^{y} \frac{\bar{\mu}_{1}-\bar{\mu}(z / N)}{\mu_{1}+\bar{\mu}(z / N)} \\
& =1+\sum_{y=1}^{N-1} \frac{1}{\left(\begin{array}{c}
N-1 \\
y
\end{array}\right)} \prod_{z=1}^{y} \frac{N\left(\bar{\mu}_{1} / \bar{\mu}\right)-z}{N\left(\mu_{1} / \bar{\mu}\right)+z} \\
& =1+\sum_{y=1}^{N-1} \frac{1}{\left(\begin{array}{c}
N-1 \\
y
\end{array}\right)} \frac{\Gamma\left(A_{N}\right)}{\Gamma\left(A_{N}-y\right)} \frac{\Gamma\left(B_{N}+1\right)}{\Gamma\left(B_{N}+1+y\right)} \\
& =: 1+\sum_{y=1}^{N-1} \frac{1}{\left(\begin{array}{c}
N-1 \\
y
\end{array}\right)} \psi_{N}(y) .
\end{aligned}
$$

Proceeding as before, we have

$$
\begin{aligned}
& 1+\frac{\psi_{N}(1)}{N-1}+\psi_{N}(N-1) \\
& <\varphi(N)<1+\frac{\psi_{N}(1)}{N-1} \\
& \quad+\psi_{N}(N-1)+O\left(N^{-2}\right),
\end{aligned}
$$

showing that $\varphi(N) \underset{N \text { large }}{\sim} 1+\psi_{N}(1) /(N-1)+\psi_{N}(N-1)$. The dominating term in $\varphi(N)$ is thus either the one corresponding to $y=1$ or the one corresponding to $y=N-1$. get

With $r:=\left(\left(\bar{\mu}_{1}^{\mu_{1}} / \bar{\mu}_{2}^{\bar{\mu}_{2}}\right)\left(\mu_{1}^{\mu_{1}} / \mu_{2}^{\mu_{2}}\right)\right)^{1 / \bar{\mu}}$, by Stirling formula, we

$$
\begin{aligned}
\varphi(N) \underset{N \text { large }}{\sim} 1 & +\frac{1}{N-1} \frac{A_{N}-1}{B_{N}+1} \\
& +\frac{\Gamma\left(A_{N}\right)}{\Gamma\left(A_{N}-N+1\right)} \frac{\Gamma\left(B_{N}+1\right)}{\Gamma\left(B_{N}+N\right)} \sim 1 \\
& +\frac{1}{N-1} \frac{v_{2}+1}{v_{1}} \\
& +\frac{\Gamma\left(N\left(v_{2}+1\right)\right)}{\Gamma\left(N v_{2}+1\right)} \frac{\Gamma\left(N \nu_{1}+1\right)}{\Gamma\left(N\left(\nu_{1}+1\right)\right)} \\
= & \frac{1}{N-1} \frac{v_{2}+1}{v_{1}} \\
+ & \frac{v_{1}+1}{v_{2}+1} \frac{\left(\begin{array}{c}
N\left(v_{2}+1\right) \\
N v_{2}
\end{array}\right)}{\left(\begin{array}{c}
N\left(v_{1}+1\right) \\
N v_{1}
\end{array}\right)}
\end{aligned}
$$




$$
\begin{aligned}
& \underset{N \text { large }}{\sim} 1+\frac{1}{N-1} \frac{\nu_{2}+1}{v_{1}} \\
& +\sqrt{\frac{\nu_{1}+1}{v_{2}+1}}\left(\frac{\left(\nu_{2}+1\right)^{\nu_{2}+1}}{v_{2}^{\nu_{2}}} \frac{v_{1}^{\nu_{1}}}{\left(\nu_{1}+1\right)^{\nu_{1}+1}}\right)^{N} \\
& =: 1+\frac{1}{N-1} \frac{v_{2}+1}{v_{1}}+\sqrt{\frac{v_{1}+1}{v_{2}+1}} r^{N} .
\end{aligned}
$$

Now, whenever $\mu_{1}<\mu_{2}$, regardless of whether $\mu>1$ or $\mu<1$,

$$
r:=\frac{\left(\nu_{2}+1\right)^{\nu_{2}+1}}{\nu_{2}^{\nu_{2}}} \frac{v_{1}^{\nu_{1}}}{\left(\nu_{1}+1\right)^{\nu_{1}+1}}=\left(\frac{\bar{\mu}_{1}^{\bar{\mu}_{1}}}{\bar{\mu}_{2}^{\bar{\mu}_{2}}} \frac{\mu_{1}^{\mu_{1}}}{\mu_{2}^{\mu_{2}}}\right)^{1 / \bar{\mu}}>1 .
$$

Indeed, let $H\left(\mu_{1}\right)=-\left(\mu_{1} \log \mu_{1}+\bar{\mu}_{1} \log \bar{\mu}_{1}\right)$. The condition $r>1$ is also

$$
\frac{1}{\bar{\mu}}\left(H\left(\mu_{2}\right)-H\left(\mu_{1}\right)\right)>0
$$

If $\mu_{1}<\mu_{2}$, then $H\left(\mu_{2}\right)-H\left(\mu_{1}\right)<0$ if and only if $\mu_{2}>\bar{\mu}_{1}$ (or $\bar{\mu}<0)$ and then $(1 / \bar{\mu})\left(H\left(\mu_{2}\right)-H\left(\mu_{1}\right)\right)>0$.

If $\mu_{1}<\mu_{2}$, then $H\left(\mu_{2}\right)-H\left(\mu_{1}\right)>0$ if and only if $\mu_{2}<\bar{\mu}_{1}$ (or $\bar{\mu}>0)$ and then $(1 / \bar{\mu})\left(H\left(\mu_{2}\right)-H\left(\mu_{1}\right)\right)>0$.

We obtained the following proposition.

Proposition 6. When $\mu \neq 1$, the fixation probability also satisfies the following sharp threshold property.

(i) If $\mu_{1}<\mu_{2}$, the fixation probability tends to 0 geometrically fast because $\varphi(N) \underset{\text { Niarge }}{\sim} \sqrt{\left(\nu_{1}+1\right) /\left(\nu_{2}+1\right)} r^{N}=$ $\sqrt{\bar{\mu}_{2} / \bar{\mu}_{1}} r^{N}$, leading to $\mathbf{P}\left(\tau_{1, N}<\tau_{1,0}\right)=1 / \varphi(N)_{\text {Nlarge }}^{\sim}$ $\sqrt{\bar{\mu}_{1} / \bar{\mu}_{2}} r^{-N}$. The fixation probability of a mutant tends to 0 , algebraically fast at rate $r$ given by (63).

(ii) If $\mu_{1}>\mu_{2}$, regardless of whether $\mu>1$ or $\mu<$ $1, r<1$. Thus, $\varphi(N) \underset{\text { Niarge }}{\sim} 1+(1 / N)\left(\left(\nu_{2}+\right.\right.$ $\left.1) / \nu_{1}\right)=1+(1 / N)\left(\bar{\mu}_{1} / \mu_{1}\right)$ leading to $\mathbf{P}\left(\tau_{1, N}<\tau_{1,0}\right)=$ $1 / \varphi(N) \underset{\text { Nlarge }}{\sim} 1 /\left(1+(1 / N)\left(\bar{\mu}_{1} / \mu_{1}\right)\right) \rightarrow 1$ from below.

(iii) If $\mu_{1}=\mu_{2}$ (balanced mutations), $\nu_{1}=\nu_{2}, r=1$, and therefore, $\varphi(N) \underset{\text { Niarge }}{\sim} 2+(1 / N)\left(\bar{\mu}_{1} / \mu_{1}\right) \rightarrow 2_{+}$: the fixation probability tends to 1/2 from below.

(i) For the Moran model with selection mechanism $p(u)=$ $(1+s) u /(1+s u)$ as in (56), gene $A_{1}$ (resp., $\left.A_{2}\right)$ has fitness $w_{1}=1+s$ (resp., $\left.w_{2}=1\right)$ so that, with $w:=w_{1} / w_{2}=1+s$, the mutant relative fitness

$$
\begin{aligned}
\varphi(x) & =1+\sum_{y=1}^{x-1} \frac{1}{\left(\begin{array}{c}
N-1 \\
y
\end{array}\right)} \prod_{z=1}^{y} \frac{\bar{p}(z / N)}{p(z / N)} \\
& =1+\sum_{y=1}^{x-1} w^{-y}=\frac{1-w^{-x}}{1-w^{-1}},
\end{aligned}
$$

showing that: $\mathbf{P}\left(\tau_{1, N}<\tau_{1,0}\right)=1 / \varphi(N)=\left(1-w^{-1}\right) /\left(1-w^{-N}\right)$. Whenever $s>0(s<0)$, the mutant gene $A_{1}$ is selectively advantageous (deleterious) compared to $A_{2}$. When $w^{-1}<1$ $(s>0), \mathbf{P}\left(\tau_{1, N}<\tau_{1,0}\right)$ tends to $1-w^{-1}$, whereas when $w^{-1}>$ $1(s<0)$, this probability tends to 0 exponentially fast like $\left(w^{-1}-1\right) w^{N}$. When $s=0$ (neutral case), $\mathbf{P}\left(\tau_{1, N}<\tau_{1,0}\right)=1 / N$ tends to 0 much slower.

In this pure selection case, these results are well known (see, e.g., (3.66) page 109 of [14] and also [1] in [17]).

(ii) For the Moran model with bias mechanism (55) involving both mutation and selection,

$$
\begin{aligned}
\varphi(x) & =1+\sum_{y=1}^{x-1} \frac{1}{\left(\begin{array}{c}
N-1 \\
y
\end{array}\right)} \prod_{z=1}^{y} \frac{\bar{p}(z / N)}{p(z / N)} \\
& =1+\sum_{y=1}^{x-1} \frac{1}{\left(\begin{array}{c}
N-1 \\
y
\end{array}\right)} \psi_{N}(y),
\end{aligned}
$$

where, with $\tilde{\mu}=(1+s) \bar{\mu}_{2}-\mu_{1}=\bar{\mu}+s \bar{\mu}_{2}, A_{N}=N\left(\bar{\mu}_{1} / \widetilde{\mu}\right), B_{N}=$ $N\left(\mu_{1} / \widetilde{\mu}\right), \psi_{N}(y)=\left(\Gamma\left(A_{N}\right) / \Gamma\left(A_{N}-y\right)\right)\left(\Gamma\left(B_{N}+1\right) / \Gamma\left(B_{N}+1+\right.\right.$ $y))$.

From the preceding analysis, we conclude that with

$$
\begin{aligned}
& r:=\left(\frac{\bar{\mu}_{1}^{\bar{\mu}_{1}}}{\left(\bar{\mu}_{2}(1+s)\right)^{\bar{\mu}_{2}(1+s)}} \frac{\mu_{1}^{\mu_{1}}}{\left(1-\bar{\mu}_{2}(1+s)\right)^{1-\bar{\mu}_{2}(1+s)}}\right)^{1 /\left[\bar{\mu}+s \bar{\mu}_{2}\right]}, \\
& \varphi(N) \underset{N \text { large }}{\sim} 1+\frac{1}{N-1} \frac{\bar{\mu}_{1}}{\mu_{1}}+\sqrt{(1+s) \frac{\bar{\mu}_{2}}{\bar{\mu}_{1}}} r^{N} .
\end{aligned}
$$

We thus obtained the following proposition.

Proposition 7. For the Moran model with bias mechanism (55):

(i) if $w=1+s<\bar{\mu}_{1} / \bar{\mu}_{2}$, the fixation probability tends to 0 geometrically fast because $\varphi(N)$ N $\tilde{N}^{\text {large }}$ $\sqrt{w\left(\bar{\mu}_{2} / \bar{\mu}_{1}\right)} r^{N}$, leading to $\mathbf{P}\left(\tau_{1, N}<\tau_{1,0}\right)=1 / \varphi(N)$ N $\sim_{\text {large }}\left(1 / \sqrt{w\left(\bar{\mu}_{2} / \bar{\mu}_{1}\right)}\right) r^{-N}$. The fixation probability of a mutant tends to 0 , algebraically fast at rate $r$ now given by (67);

(ii) if $w>\bar{\mu}_{1} / \bar{\mu}_{2}, \varphi(N) \underset{\text { Narge }}{\sim} 1+(1 / N)\left(\bar{\mu}_{1} / \mu_{1}\right)$ leading to $\mathbf{P}\left(\tau_{1, N}<\tau_{1,0}\right)=1 / \varphi(N) \underset{\text { Niarge }}{\sim} 1 /\left(1+(1 / N)\left(\bar{\mu}_{1} /\right.\right.$ $\left.\left.\mu_{1}\right)\right) \rightarrow 1$ from below;

(iii) in the critical regime when $w=\bar{\mu}_{1} / \bar{\mu}_{2}$ and then $\varphi(N) \underset{\text { Narge }}{\sim} 2+(1 / N)\left(\bar{\mu}_{1} / \mu_{1}\right)$, the fixation probability tends again to $1 / 2$.

Proof. Regardless of whether $\mu<1$ or $\mu>1$, the extinction condition $r>1$ is achieved whenever

$$
\frac{1}{\bar{\mu}+s \bar{\mu}_{2}}\left(H\left(1-\bar{\mu}_{2}(1+s)\right)-H\left(\mu_{1}\right)\right)>0 .
$$


By the above arguments, this leads to the following mutationselection balance condition: $\mu_{1}<1-\bar{\mu}_{2}(1+s)$ which is also

$$
w:=1+s<\frac{\bar{\mu}_{1}}{\bar{\mu}_{2}}
$$

The selective advantage of allele $A_{1}$ (its relative fitness) should not be too large, and the upper bound is given by the ratio of the mutation probabilities $\bar{\mu}_{1} / \bar{\mu}_{2}$. Note that $\mu_{1}<\mu_{2}$ (resp., $\left.\mu_{1}>\mu_{2}\right) \Rightarrow \bar{\mu}_{1} / \bar{\mu}_{2}>1$ (resp., $\bar{\mu}_{1} / \bar{\mu}_{2}<1$ ). In the presence of selection, the extinction condition can be achieved even if $\mu_{1}>\mu_{2}$, provided that $s<0$ is small enough. When $\mu_{1}=\mu_{2}$, the extinction of the mutant allele occurs if it is selectively deleterious: $s<0$.

To the best of the author's knowledge, the results displayed in Propositions 5-7 seem to be new.

3.4. Spectral Representation and Invariant Measure of the Moran Model with Mutations. Except for some exceptional special cases, the spectral measure associated to the Moran model with general bias mechanism $p$ as in (44) is not known. One of the notable exceptions is the Moran model with positive mutation probabilities $\mu_{1}, \mu_{2}$; see [18-20]. The transition matrix $P$ of this model has therefore the $\mathrm{BD}$ structure (52).

For the Moran model with mutations, the orthogonal polynomials coincide with the dual Hahn polynomials $R_{x}\left(\lambda_{k}\right):=H_{k}(x)$, where, with $\lambda_{k}=k(k+N v-1)$,

$$
H_{k}(x)={ }_{3} F_{2}\left(\left(\begin{array}{c}
-k, k+N v-1,-x \\
N v_{1},-N
\end{array}\right) ; 1\right), \quad k=0, \ldots, N
$$

are the classical Hahn or Hahn-Eberlein polynomials. We note that (Hahn case) $\mu<1 \Rightarrow \nu_{1}, \nu_{2}>0$ with $\nu_{1}, v_{2} \rightarrow+\infty$ $\left(\mu \rightarrow 1^{-}\right)$and $\nu_{1}, \nu_{2} \rightarrow 0\left(\mu \rightarrow 0^{+}\right)$, whereas (HahnEberlein case) $\mu>1 \Rightarrow \nu_{1}, \nu_{2}<-1$ with $\nu_{1}, \nu_{2} \rightarrow-\infty$ $\left(\mu \rightarrow 1^{+}\right)$and $\nu_{1}, \nu_{2} \rightarrow-1\left(\mu \rightarrow 2^{-}\right)$.

The case $\mu<1(\mu>1)$ corresponds to a weak (strong) mutation regime.

As a truncated hypergeometric series, the polynomials $R_{x}\left(\lambda_{k}\right)$ are of degree $k$ in $x$. In this case, thanks to the 3term recurrence satisfied by the $H_{k}(x)$, the eigenvalues of the transition matrix (52) are

$$
t_{k}=1-\frac{\lambda_{k}}{N(N+N \nu)}=1-\frac{k}{N}\left(\mu+\frac{k-1}{N} \bar{\mu}\right)
$$

depending only on the total mutation pressure $\mu$. In particular, $1-t_{1}=\mu / N$ is the spectral gap, and $t_{N}=\bar{\mu} / N$ is the smallest eigenvalue (with $t_{N}<0$ if $\mu>1$ ).

(i) In the weak mutation regime $\mu<1$, the RW is spectrally positive with

$$
0<t_{N}=\frac{\bar{\mu}}{N}<\cdots<t_{1}=1-\frac{\mu}{N}<t_{0}=1
$$

(ii) In the strong mutation regime $1<\mu<2$, the RW is not spectrally positive with

$$
t_{N}=\frac{\bar{\mu}}{N}<\cdots<0<\cdots<t_{1}=1-\frac{\mu}{N}<t_{0}=1 .
$$

When $N$ gets large, there is a fixed (independent of $N$ ) number of negative eigenvalues. Only when $\mu=2$ do we have a number of negative eigenvalues of order $\sqrt{N / 2}$.

Invariant Measure. Furthermore, with $(a)_{x}:=\Gamma(a+x) / \Gamma(a)$ the rising factorial of $a$, the invariant probability measure is the generalized bivariate hypergeometric distribution:

$$
\pi_{x}=\left(\begin{array}{c}
N \\
x
\end{array}\right) \frac{\left(N v_{1}\right)_{x}\left(N v_{2}\right)_{N-x}}{(N v)_{N}}, \quad x=0, \ldots, N
$$

which may also be recast as

$$
\pi_{x}=\frac{\left(\begin{array}{c}
-N v_{1} \\
x
\end{array}\right)\left(\begin{array}{c}
-N v_{2} \\
N-x
\end{array}\right)}{\left(\begin{array}{c}
-N v \\
N
\end{array}\right)}, \quad x=0, \ldots, N
$$

with the convention $\left(\begin{array}{c}-a \\ x\end{array}\right)=\{-a\}_{x} / x !,\{-a\}_{x}=-a(-a-$ 1) $\cdots(-a-x+1)$, the falling factorials of $-a$ with $\{-a\}_{x}=$ $(-1)^{x}(a)_{x}$.

The mean and variance of $\pi_{x}$ are

$$
\begin{gathered}
m_{\infty}:=N \frac{\nu_{1}}{\nu}=N \frac{\mu_{1}}{\mu}, \\
\sigma_{\infty}^{2}:=N^{2} \frac{\nu_{1} \nu_{2}}{\nu^{2}} \frac{1+\nu}{N \nu+1}=\frac{N^{2}}{N \mu+\bar{\mu}} \frac{\mu_{1} \mu_{2}}{\mu^{2}} .
\end{gathered}
$$

Upon scaling, the law of $U_{\infty}:=X_{\infty} / N$ is very much peaked around its mean $\mu_{1} / \mu$, with density

$$
f_{N}(u)=N \pi_{N u}=\frac{e^{N V(u)}}{\int_{0}^{1} d u e^{N V(u)}}, \quad u \in(0,1)
$$

where $V(u)=\log W(u)$ and, by Stirling formula,

$$
\begin{aligned}
& W(u) \stackrel{(\mu<1)}{=} \frac{\left(\nu_{1}+u\right)^{\nu_{1}+u}}{v_{1}^{\nu_{1}} u^{u}} \frac{\left(\nu_{2}+1-u\right)^{\nu_{2}+1-u}}{v_{2}^{\nu_{2}}(1-u)^{1-u}} \\
& \times \frac{v^{\nu}}{(\nu+1)^{\nu+1}}, \quad \text { if } \nu_{1}, \nu_{2}>0, \\
& W(u) \stackrel{(\mu>1)}{=} \frac{\left(-v_{1}\right)^{-v_{1}}}{u^{u}\left(-v_{1}-u\right)^{-v_{1}-u}} \\
& \times \frac{\left(-v_{2}\right)^{-v_{2}}}{(1-u)^{1-u}\left(-v_{2}-1+u\right)^{-v_{2}-1+u}} \\
& \times \frac{(-v-1)^{-v-1}}{(-v)^{-v}} \text { if } v_{1}, v_{2}<-1, \\
& W(u) \stackrel{(\mu=1)}{=} \frac{\mu_{1}^{u} \bar{\mu}_{1}^{1-u}}{u^{u}(1-u)^{1-u}} .
\end{aligned}
$$


We have $V^{\prime}(u)=0$ at $u_{*}=\nu_{1} / \nu=\mu_{1} / \mu$, and $V^{\prime \prime}\left(u_{*}\right)=$ $\left(-\nu^{2} /(1+\nu)\right)\left(1 / \nu_{1}+1 / \nu_{2}\right)=-\mu^{3} / \mu_{1} \mu_{2}<0$.

A saddle point estimate gives

$$
\int_{0}^{1} d u e^{N V(u)}=e^{N V\left(u_{*}\right)} \sqrt{\frac{2 \pi}{N\left|V^{\prime \prime}\left(u_{*}\right)\right|}},
$$

$$
\text { and so } f_{N}(u) \sim \sqrt{\frac{N\left|V^{\prime \prime}\left(u_{*}\right)\right|}{2 \pi}} e^{-N\left(V\left(u_{*}\right)-V(u)\right)} \text {. }
$$

(i) When both $\nu_{1}, v_{2}>0(\mu<1)$, the distribution (74) is the negative hypergeometric distribution which can be obtained as a beta $\left(N v_{1}, N v_{2}\right)$ mixture of the binomial distribution $\left(\begin{array}{c}N \\ x\end{array}\right) p^{x}(1-p)^{N-x}$ with parameter $p \in[0,1]$, namely,

$$
\begin{aligned}
\pi_{x}= & \left(\begin{array}{c}
N \\
x
\end{array}\right) \int_{0}^{1} p^{x}(1-p)^{N-x} \frac{\Gamma(N \nu)}{\Gamma\left(N v_{1}\right) \Gamma\left(N v_{2}\right)} \\
& \times p^{N v_{1}-1}(1-p)^{N v_{2}-1} d p,
\end{aligned}
$$

with (by Stirling formula)

$$
\begin{aligned}
\pi_{0} & =\frac{\Gamma(N \nu)}{\Gamma\left(N v_{1}\right) \Gamma\left(N v_{2}\right)} \int_{0}^{1} p^{N v_{1}-1}(1-p)^{N\left(v_{2}+1\right)-1} d p \\
& =\frac{\Gamma(N \nu)}{\Gamma\left(N v_{2}\right)} \frac{\Gamma\left(N\left(v_{2}+1\right)\right)}{\Gamma(N(v+1))} \\
& \sim \sqrt{\frac{\nu_{2}}{\nu} \frac{\nu+1}{\nu_{2}+1}}\left(\frac{\left(\nu_{2}+1\right)^{\nu_{2}+1}}{v_{2}^{\nu_{2}}} \frac{\nu^{\nu}}{(\nu+1)^{\nu+1}}\right)^{N} \\
& =: \sqrt{\frac{\mu_{2}}{\mu \bar{\mu}_{1}}} \rho^{N},
\end{aligned}
$$

displaying exponential decay $\left(\rho=\left(\bar{\mu}_{1}^{\bar{\mu}_{1}} \mu^{\mu} / \mu_{2}^{\mu_{2}}\right)^{1 / \bar{\mu}}<1\right)$. Note that, with

$$
\bar{\rho}:=\frac{\left(\nu_{1}+1\right)^{\nu_{1}+1}}{\nu_{1}^{\nu_{1}}} \frac{v^{\nu}}{(\nu+1)^{\nu+1}}=\left(\frac{\bar{\mu}_{2}^{\bar{\mu}_{2}} \mu^{\mu}}{\mu_{1}^{\mu_{1}}}\right)^{1 / \bar{\mu}}<1,
$$

it holds that

$$
\begin{aligned}
\pi_{N} & =\frac{\Gamma(N \nu)}{\Gamma\left(N \nu_{1}\right) \Gamma\left(N v_{2}\right)} \int_{0}^{1} p^{N\left(v_{1}+1\right)-1}(1-p)^{N v_{2}-1} d p \\
& =\frac{\Gamma(N \nu)}{\Gamma\left(N v_{1}\right)} \frac{\Gamma\left(N\left(\nu_{1}+1\right)\right)}{\Gamma(N(v+1))} \sim \sqrt{\frac{\mu_{1}}{\mu \bar{\mu}_{2}}} \bar{\rho}^{N} .
\end{aligned}
$$

Note that both $\rho$ and $\bar{\rho}<1$ because, with $h(x):=-x \log x$, $\mu<1 \Rightarrow h(\mu)>h\left(\mu_{2}\right)-h\left(\bar{\mu}_{1}\right)$ and $h(\mu)>h\left(\mu_{1}\right)-h\left(\bar{\mu}_{2}\right)$.

Furthermore, the rate $r$ (63) appearing in the fixation probability is $r=\rho / \bar{\rho}$.

The case of symmetric mutations $\mu_{1}=\mu_{2}$ (with $\mu<1$ ) is obtained while setting $\nu_{1}=\nu_{2}=\nu / 2$.

(ii) When both $\nu_{1}, v_{2}<-1(2>\mu>1)$, the distribution $\pi_{x}$ in (75) constitutes the classical (generalized) hypergeometric distribution arising in sampling without replacement.
By Stirling formula, for all $b>a>0$ (exponential growth),

$$
\left(\begin{array}{l}
N b \\
N a
\end{array}\right) \sim \frac{1}{\sqrt{2 \pi(b-a) N / b}}\left[\frac{b^{b}}{a^{a}(b-a)^{b-a}}\right]^{N} .
$$

This shows that, with $\rho<1$ and $\bar{\rho}<1$,

$$
\begin{aligned}
& \pi_{0}=\frac{\left(\begin{array}{c}
-N v_{2} \\
N
\end{array}\right)}{\left(\begin{array}{c}
-N v \\
N
\end{array}\right)} \\
& \sim \sqrt{\frac{\nu_{2}(\nu+1)}{\nu\left(\nu_{2}+1\right)}}\left[\frac{\left(-\nu_{2}\right)^{-\nu_{2}}}{\left(-\nu_{2}-1\right)^{-\nu_{2}-1}} \frac{(-\nu-1)^{-\nu-1}}{(-\nu)^{-\nu}}\right]^{N} \\
& =: \sqrt{\frac{\mu_{2}}{\mu \bar{\mu}_{1}}} \rho^{N}, \\
& \pi_{N}=\frac{\left(\begin{array}{c}
-N v_{1} \\
N
\end{array}\right)}{\left(\begin{array}{c}
-N \nu \\
N
\end{array}\right)} \\
& \sim \sqrt{\frac{\nu_{1}(\nu+1)}{\nu\left(\nu_{1}+1\right)}}\left[\frac{\left(-v_{1}\right)^{-\nu_{1}}}{\left(-\nu_{1}-1\right)^{-\nu_{1}-1}} \frac{(-\nu-1)^{-\nu-1}}{(-\nu)^{-\nu}}\right]^{N} \\
& =: \sqrt{\frac{\mu_{1}}{\mu \bar{\mu}_{2}}} \bar{\rho}^{N} .
\end{aligned}
$$

Note that again

$$
\rho=\left(\frac{\bar{\mu}_{1}^{\bar{\mu}_{1}} \mu^{\mu}}{\mu_{2}^{\mu_{2}}}\right)^{1 / \bar{\mu}}, \quad \bar{\rho}=\left(\frac{\bar{\mu}_{2}^{\bar{\mu}_{2}} \mu^{\mu}}{\mu_{1}^{\mu_{1}}}\right)^{1 / \bar{\mu}},
$$

with $\rho$ and $\bar{\rho}<1$ because, with $h(x):=-x \log x, \mu>1 \Rightarrow$ $h(\mu)<h\left(\mu_{2}\right)-h\left(\bar{\mu}_{1}\right)$ and $h(\mu)<h\left(\mu_{1}\right)-h\left(\bar{\mu}_{2}\right)$.

(iii) When both $\nu_{1}, \nu_{2}=-1\left(\mu_{1}=\mu_{2}=1\right)$, we get the standard hypergeometric distribution $\pi_{x}=\left(\begin{array}{c}N \\ x\end{array}\right)\left(\begin{array}{c}N \\ N-x\end{array}\right) /\left(\begin{array}{c}2 N \\ N\end{array}\right)$, with $\pi_{0}=\pi_{N}=1 /\left(\begin{array}{c}2 N \\ N\end{array}\right) \sim \sqrt{\pi N} 4^{-N}$.

The case of symmetric mutations $\mu_{1}=\mu_{2}$ (with $\mu>1$ ) is obtained while setting $\nu_{1}=\nu_{2}=\nu / 2$.

(iv) When $\mu_{1}=\mu_{2}=1$ (Bernoulli-Laplace), the transition probabilities read $q_{x}=(x / N)^{2}, r_{x}=2(x / N)(1-x / N), p_{x}=$ $(1-x / N)^{2}$. Here, $\pi_{x}=\left(\begin{array}{l}N \\ x\end{array}\right)\left(\begin{array}{c}N \\ N-x\end{array}\right) /\left(\begin{array}{c}2 N \\ N\end{array}\right)$ (the standard hypergeometric distribution), $\mu_{k}=((2 N+1-2 k) /(2 N+$ $1-k))\left(\begin{array}{c}N \\ k\end{array}\right) /\left(\begin{array}{c}2 N-k \\ N\end{array}\right)$, and $t_{k}=1-\left(k / N^{2}\right)(2 N+1-k)$.

The expected return time to $\{0\}$ is $2^{2 N} / \sqrt{\pi N}$, which is huge when $N$ is large, whereas the expected return time to $\{N / 2\}$ is of order $\sqrt{\pi N} / 2$, much smaller.

(v) On the critical line $\mu_{1}+\mu_{2}=1, p(u)=\mu_{1}$ is constant and so the transition probabilities become affine functions of the state: $q_{x}=\bar{\mu}_{1}(x / N), \quad r_{x}=\bar{\mu}_{1}+\left(2 \mu_{1}-1\right)(x / N)$, and $p_{x}=$ $\mu_{1}(1-x / N)$. Here, $\pi_{x}=\left(\begin{array}{l}N \\ x\end{array}\right) \mu_{1}^{x} \bar{\mu}_{1}^{N-x}, \mu_{k}=\left(\begin{array}{c}N \\ k\end{array}\right) \mu_{1}^{k} \bar{\mu}_{1}^{N-k}$ are binomial $\operatorname{bin}\left(N, \mu_{1}\right)$-distributed and self-dual, and $t_{k}=$ $1-k / N$, independent of $\mu_{1}$ (this RW is spectrally nonnegative). The Hahn polynomials boil down to $H_{k}(x)=$ ${ }_{2} F_{1}\left(\left(\begin{array}{c}-k,-x \\ -N\end{array}\right) ; \mu_{1}^{-1}\right), k=0, \ldots, N$, a class of Krawtchouk polynomials. When $\mu_{1}=1 / 2$ (the lazy Ehrenfest urn), 
the holding probabilities are $r_{x}=1 / 2$, and both $\pi_{x}$ and $\mu_{k}$ are symmetric $\operatorname{bin}(N, 1 / 2)$-distributed.

Spectral Measure When $\mu \neq 1$. The spectral probability measure is

$\mu_{k}=\frac{2 k+N v-1}{k+N v-1}\left(\begin{array}{c}N \\ k\end{array}\right) \frac{\left(N v_{2}\right)_{N}}{(k+N v)_{N}} \frac{\left(N v_{1}\right)_{k}}{\left(N v_{2}\right)_{k}}, \quad k=0, \ldots, N$,

and the orthogonality relation reads

$$
\begin{aligned}
\sum_{k=0}^{N} \mu_{k} R_{x}\left(\lambda_{k}\right) R_{y}\left(\lambda_{k}\right) \\
=\frac{\left(N v_{2}\right)_{N}}{N !} \frac{x !(N-x) !}{\left(N v_{1}\right)_{x}\left(N v_{2}\right)_{N-x}} \delta_{x, y} \\
=\frac{\pi_{0}}{\pi_{x}} \delta_{x, y} .
\end{aligned}
$$

Notice that, in our notations: $\mathfrak{q}_{x}\left(t_{k}\right)=R_{x}(N(N+N v)(1-$ $\left.\left.t_{k}\right)\right)=R_{x}\left(N^{2}\left(1-t_{k}\right) /(\bar{\mu})\right)$. In particular,

$$
\begin{gathered}
\mathfrak{q}_{x}\left(t_{1}\right)=: H_{1}(x)=1-\frac{v}{N \nu_{1}} x, \\
\mathfrak{q}_{x}\left(t_{2}\right)=: H_{2}(x)=1-A x+B x^{2},
\end{gathered}
$$

where

$$
\begin{gathered}
A=\frac{N v+1}{N v_{1}} \frac{2 N v_{1}+2+v_{2}-v_{1}}{(N-1)\left(N v_{1}+1\right)}, \\
B=\frac{(N v+1)(N v+2)}{N^{2}\left(N v_{1}+1\right)(N-1) v_{1}} .
\end{gathered}
$$

Evolution of the Conditional Mean and Variance of $X_{n}$ given $X_{0}=x . \mathfrak{q}_{x}\left(t_{1}\right)$ and $\mathfrak{q}_{x}\left(t_{2}\right)$ being right eigenvectors of $P$,

$$
\begin{aligned}
& \mathbf{E}_{x} H_{1}\left(X_{n}\right)=t_{1}^{n} H_{1}(x), \\
& \mathbf{E}_{x} H_{2}\left(X_{n}\right)=t_{2}^{n} H_{2}(x)
\end{aligned}
$$

leading, by virtue of (25), to the following peoposition.

Proposition 8. For the Moran model with mutations

$$
\begin{aligned}
\mathbf{E}_{x}\left(X_{n}\right)= & m_{\infty}\left(1-t_{1}^{n} H_{1}(x)\right) \underset{n \rightarrow \infty}{\longrightarrow} m_{\infty} \\
= & N \frac{\nu_{1}}{\nu}=N \frac{\mu_{1}}{\mu}, \\
\sigma_{x}^{2}\left(X_{n}\right)= & \frac{A m_{\infty}}{B}\left(1-t_{1}^{n} H_{1}(x)\right) \\
& -m_{\infty}^{2}\left(1-t_{1}^{n} H_{1}(x)\right)^{2} \\
& -\frac{1}{B}+\frac{1}{B} t_{2}^{n} H_{2}(x) \underset{n \rightarrow \infty}{\longrightarrow} \sigma_{\infty}^{2} .
\end{aligned}
$$

This shows how the mean and variance of $X_{n}$ vary, during the course of evolution, as a function of $n$ and the initial condition $X_{0}=x$ before reaching the mean and variance of the limit law. Concerning the mean value

$$
\mathbf{E}_{x}\left(X_{n}\right)-m_{\infty}=t_{1}^{n}\left(x-m_{\infty}\right)
$$

and depending on $x>m_{\infty}$ or $x<m_{\infty}, \mathbf{E}_{x}\left(X_{n}\right)$ approaches monotonically the limiting value $m_{\infty}$ from above or from below. Note however that for all initial conditions $x$,

$$
\left|\mathbf{E}_{x}\left(X_{n}-m_{\infty}\right)\right| \text { is a decreasing (negentropic) function }
$$

of $n$.

Let us now consider the variance. Noting that $A / B-2 m_{\infty}=$ $\left(\mu_{1}-\mu_{2}\right)(2-\mu) /(\mu-1)$, rearranging the terms,

$$
\begin{aligned}
\sigma_{x}^{2}\left(X_{n}\right)= & \sigma_{\infty}^{2}-m_{\infty} H_{1}(x) \frac{\left(\mu_{2}-\mu_{1}\right)(2-\mu)}{\bar{\mu}} t_{1}^{n} \\
& -m_{\infty}^{2} H_{1}(x)^{2} t_{1}^{2 n}+\frac{1}{B} H_{2}(x) t_{2}^{n}
\end{aligned}
$$

with $\left(\mu_{2}-\mu_{1}\right)(2-\mu) / \bar{\mu}>0$ if $\left(\mu_{2}>\mu_{1}\right.$ and $\left.\mu<1\right)$ or $\left(\mu_{2}<\mu_{1}\right.$ and $\left.\mu>1\right)$ and $t_{2}<t_{1}^{2}<t_{1}<1$. The corrective term to the limiting variance is a linear combination of three terms with geometric decay at different rates. For large $n$, the discrepancy between $\sigma_{x}^{2}\left(X_{n}\right)$ and $\sigma_{\infty}^{2}$ is indeed controlled by the slower $t_{1}^{n}$ term. In general, the variance does not increase monotonically towards its limiting value, starting from $\sigma_{x}^{2}\left(X_{0}=x\right)=0$.

Proposition 9. If for instance starting from $x<N \mu_{1} / \mu=$ $m_{\infty}$ (which is $\left.H_{1}(x)>0\right)$, depending on $\left(\mu_{2}-\mu_{1}\right)(2-\mu) / \bar{\mu}>0$ or not, $\sigma_{x}^{2}\left(X_{n}\right)$ approaches its limiting value $\sigma_{\infty}^{2}$ from below or from above, respectively. When $\left(\mu_{2}-\mu_{1}\right)(2-\mu) / \bar{\mu}<0$, there is a time $n=n_{*}(x)$ at which the fluctuation $\sigma_{x}^{2}\left(X_{n}\right)$ is maximum, and this maximum exceeds $\sigma_{\infty}^{2}$. The conclusions should be reversed would $x>N \mu_{1} / \mu=m_{\infty}$ (or $\left.H_{1}(x)<0\right)$.

It can also be shown [21, page 47] that, for $m \geq 0$,

$$
\operatorname{cov}_{x}\left(X_{n}, X_{n+m}\right)=t_{1}^{m} \sigma_{x}^{2}\left(X_{n}\right) \text {, }
$$

meaning a geometric asymptotic decay of the autocovariance also controlled by the second largest eigenvalue $t_{1}$.

Evolution of Heterozygosity. Let

$$
\mathfrak{h}\left(X_{n}\right)=X_{n}\left(N-X_{n}\right)=: X_{n} \bar{X}_{n}
$$

denote the heterozygosity of the chain. This function is the largest when $X_{n}=N / 2$, that is, when both type population sizes $X_{n}$ and $\bar{X}_{n}$ are the closest. The exact evolution of $\mathbf{E}_{x} \mathfrak{h}\left(X_{n}\right)$ and $\sigma_{x}^{2}\left(\mathfrak{h}\left(X_{n}\right)\right)$ can be derived in a similar way using (25). For the variance $\sigma_{x}^{2}\left(\mathfrak{h}\left(X_{n}\right)\right), \mathfrak{q}_{x}\left(t_{4}\right)=: H_{4}(x)$, as a degree-4 polynomial in $x$, is needed. It can be shown that $\mathbf{E}_{x} \mathfrak{h}\left(X_{n}\right)$ tends monotonically to $\sigma_{\infty}^{2} \mu(N-1) \sim N^{2}\left(\mu_{1} \mu_{2} / \mu^{2}\right)$, 
geometrically fast at rate $t_{1}$, as $n \rightarrow \infty$. The variance tends to 0 geometrically fast as $n \rightarrow \infty$, and it is maximal at some intermediate time $n=n_{*}(x)$.

Note that, with $\mathfrak{g}\left(X_{n}\right)=X_{n}-\bar{X}_{n}=2 X_{n}-N$ denoting the population gap,

$$
\mathbf{E}_{x} \mathfrak{g}\left(X_{n}\right)=2 m_{\infty}\left(1-t_{1}^{n} H_{1}(x)\right)-N
$$

and this function, just like $\mathbf{E}_{x}\left(X_{n}\right)-m_{\infty}$, is not always (i.e., independently of $x$ ) decreasing with $n$.

Increase of the Conditional Entropy Given $X_{0}=x(H$ Theorem). Let $\mathbf{P}\left(X_{n} \mid X_{0}=x\right)=P^{n}\left(x, X_{n}\right), n \geq 1$. For any initial distribution $\mathbf{P}\left(X_{0}=x\right)$, with

$$
\begin{aligned}
H_{n} & :=-\mathbf{E} \log \mathbf{P}\left(X_{n} \mid X_{0}\right) \\
& =-\sum_{x=0}^{N} \mathbf{P}\left(X_{0}=x\right) \mathbf{E} \log \mathbf{P}\left(X_{n} \mid X_{0}=x\right),
\end{aligned}
$$

$$
n \geq 1, H_{0}=0
$$

the Shannon conditional entropy of the chain, we have [22]

$$
0 \leq H_{n+1}-H_{n} \leq H_{n}-H_{n-1}
$$

The first inequality shows that $H_{n}$ is nondecreasing. It converges to its limiting maximal value, which is the Shannon entropy of the invariant measure. The second inequality shows that $H_{n}$ is $n$-concave: the speed of increase of entropy decreases as time passes by. In particular, letting $H_{n}(x):=$ $-\mathbf{E} \log \mathbf{P}\left(X_{n} \mid X_{0}=x\right)$, for all fixed starting point $x, H_{0}(x)=$ 0 and

$$
0 \leq H_{n+1}(x)-H_{n}(x) \leq H_{n}(x)-H_{n-1}(x) .
$$

The increase of entropy is a manifestation of irreversibility apparently in conflict with the reversibility property of all ergodic BD chains and their recurrence of states.

Empirical Average and Large Deviation [23]. Given $X_{0}=x_{0}$, let

$$
M_{n}^{U}:=\frac{1}{n} S_{n}^{U}:=\frac{1}{n} \sum_{m=1}^{n} U\left(X_{m}\right)
$$

denote the empirical average of some bounded measurable output function $U$ of the (ergodic) chain. Examples of interesting $U$ s are $U(x)=x$ in which case $M_{n}^{U}$ is directly the empirical average of the $X \mathrm{~s}$, but also $U(x)=\mathbf{1}(x=y)$ in which case $S_{n}^{U}$ is the fraction of time spent by the walker at state $y$ (its local time). With $\mathbf{e}_{x_{0}}^{\prime}$ the row vector which is 0 everywhere but at position $x_{0}$ where it is 1 and 1 a column vector of 1 s, we have

$$
\begin{aligned}
& \mathbf{E}\left(e^{-\beta S_{n}^{U}} \mid X_{0}=x_{0}\right) \\
& =\sum_{x_{1}, \ldots, x_{n}} e^{-\beta \sum_{m=1}^{n} U\left(x_{m}\right)} \prod_{m=1}^{n} P\left(x_{m-1}, x_{m}\right) \\
& =\sum_{x_{1}, \ldots, x_{n}, m=1} \prod_{m-1}^{n} P\left(x_{m}\right) e^{-\beta U\left(x_{m}\right)} \\
& =\mathbf{e}_{x_{0}}^{\prime} P_{\beta}^{n} \mathbf{1},
\end{aligned}
$$

where, with $\mathbf{w}_{\beta}$ a column weight-vector with $x$ entry $\mathbf{w}_{\beta}(x)=$ $e^{-\beta U(x)}, P_{\beta}:=P D_{\mathbf{w}_{\beta}}$. By Perron-Frobenius theorem, we have

$$
-\frac{1}{n} \log \mathbf{E}\left(e^{-\beta S_{n}^{U}} \mid X_{0}=x_{0}\right) \underset{n \rightarrow \infty}{\longrightarrow}-\log \lambda(\beta)=: p(\beta),
$$

where $\lambda(\beta)$ is the dominant principal eigenvalue of $P_{\beta}$ and $p(\beta)$ a concave function of $\beta \in \mathbb{R}$, with $p(0)=0$. Thus,

$$
\frac{1}{n} \log \mathbf{P}\left(\frac{1}{n} \sum_{m=1}^{n} U\left(X_{m}\right) \underset{n \rightarrow \infty}{\in} d \alpha\right) \underset{n \rightarrow \infty}{\longrightarrow} f(\alpha) d \alpha,
$$

where $f(\alpha)=\inf _{\beta}(\alpha \beta-p(\beta)) \leq 0$ is the concave Legendre transform of the pressure function $p(\beta)$, giving the large deviation rate function of $M_{n}^{U}$. Clearly,

$$
\frac{1}{n} \sum_{m=1}^{n} U\left(X_{m}\right) \underset{n \rightarrow \infty}{\stackrel{\text { a.s. }}{\longrightarrow}} \alpha_{*}:=\mathbf{E}\left(U\left(X_{\infty}\right)\right),
$$

which is the value where $f(\alpha)$ is maximum $\left(\beta_{*}=f^{\prime}\left(\alpha_{*}\right)=0\right)$ equal to 0 .

\section{A Detailed Study of the Siegmund Dual of BD Chains with an Application to the Mutation Moran Model}

In this Section, we will illustrate the power of the duality/intertwining relationship by considering the simplest Siegmund dual of a birth and death chain, with the mutation Moran birth and death example in mind. We also address here the question of computing the strong stationary time distribution that helps quantifying the "distance" to equilibrium of the original positive recurrent $\mathrm{BD}$ process.

Definition 10 (see [24]). Two discrete-time Markov processes $\left(X_{n}, \widehat{X}_{n} ; n \geq 0\right)$, with state spaces $(\mathscr{X}, \mathscr{Y})$, possibly with substochastic transition kernels, are said to be dual with respect to some nonsingular duality kernel $H \geq 0$ on the product space $(\mathscr{X}, \mathcal{Y})$ if $\forall x \in \mathscr{X}, \forall y \in \mathscr{Y}, \forall n \in \mathbb{N}$ :

$$
\mathbf{E}_{x} H\left(X_{n}, y\right)=\mathbf{E}_{y} H\left(x, \widehat{X}_{n}\right) .
$$

When state spaces $(\mathscr{X}, \mathscr{Y})=\{0, \ldots, N\}^{2}$ are finite and identical, the duality kernel is a square-matrix, and the 
transition matrix of the dual process $\widehat{X}$, say $\widehat{P}$, is obtained from the one $P$ of the direct process by

$$
\widehat{P}^{\prime}=H^{-1} P H,
$$

where $\widehat{P}^{\prime}$ stands for the transpose of $\widehat{P}$. Note that if $\widehat{P}$ is an $H$-dual to $P$, then $P$ is an $H^{\prime}$-dual to $\widehat{P}$. If $H=H^{\prime}, \widehat{P}$ is an $H$-dual to $P$, but also $P$ is an $H$-dual to $\widehat{P}$.

The Siegmund Duality Kernel. (For nonneutral population genetics models, other duality kernels have recently been shown to be of interest, see $[25,26])$. The Siegmund duality kernel is $H(x, y)=\mathbf{1}(x \leq y)$. If, for a given process $X_{n}$, a process $\widehat{X}_{n}$ exists satisfying the above condition, $\widehat{X}_{n}$ is called the Siegmund dual of $X_{n}$, see [27]. Clearly, in the BD case for $X_{n}$, the condition for the Siegmund dual to exist is that $X_{n}$ should be stochastically monotone in that, for all $y \geq 0$ and $n \geq 0, \mathbf{P}_{x}\left(X_{n}>y\right)$ should be increasing with $x$.

For positive recurrent birth and death processes, and for the Siegmund kernel, the transition matrix $\widehat{P}$ of the dual process $\widehat{X}_{n}$ reads

$$
\widehat{P}=\left[\begin{array}{ccccc}
r_{0}-q_{1} & q_{1} & & & \\
p_{1} & \widehat{r}_{1} & q_{2} & & \\
& \ddots & \ddots & \ddots & \\
& & p_{N-1} & \widehat{r}_{N-1} & q_{N} \\
& & & 0 & 1
\end{array}\right],
$$

where $\widehat{r}_{y}:=1-\left(p_{y}+q_{y+1}\right), y \in\{1, \ldots, N-1\}$ (and $\widehat{q}_{y}=p_{y}$, $\left.y=1, \ldots, N-1, \widehat{p}_{y}=q_{y+1}, y=0, \ldots, N-1\right)$. It is again the one of a BD process (but not of a Moran BD process if $P$ is a Moran transition matrix). Indeed, in this case, $H$ is an upperright triangular matrix with nonzero entries 1, whereas the nonnull entries of $H^{-1}$ are the diagonal (with entries 1) and the upper diagonal with entries -1 . The structure of $\widehat{P}$ follows from this, and the duality relation: $\widehat{P}^{\prime}=H^{-1} P H$.

For this dual to exist, we need to ensure that $p_{y}+q_{y+1} \leq 1$ for $y \in\{0, \ldots, N-1\}$ which is a necessary and sufficient condition to guarantee the stochastic monotonicity of $X_{n}$. This condition also reads: $p_{y+1}-p_{y} \geq-r_{y+1}$. The drift of $\widehat{X}_{n}$ at $y$ is

$$
\begin{aligned}
\widehat{f}(y):=\widehat{p}_{y}-\widehat{q}_{y} & =q_{y+1}-p_{y} \\
& =-f(y+1)+\left(p_{y+1}-p_{y}\right),
\end{aligned}
$$

where $f(y):=p_{y}-q_{y}$ is the drift of $X_{n}$ at $y$. We have

$$
-f(y+1)-r_{y+1} \leq \widehat{f}(y) \leq-f(y)+r_{y} .
$$

We already know that if $P$ is a spectrally nonnegative $\mathrm{BD}$ matrix, the chain is stochastically monotone. Here is another sufficient condition relative to the specific ergodic Moran case.

Proposition 11. Consider the Moran model $X_{n}$ with bias $p$ satisfying $0<p(0), p(1)<1$. If $p(u)$ is nondecreasing, the condition $p_{x}+q_{x+1} \leq 1$ is fulfilled (the Moran chain is stochastically monotone), and so the Siegmund dual exists.
Proof. We first need to guarantee that $1-p_{0}-q_{1} \geq 0$ corresponding to the row $x=0$ of $\widehat{P}$, but this is true because $p(1 / N) \geq p(0) \geq N p(0)-(N-1)$. Next, when $x=N$, the inequality clearly holds because $p_{N}=q_{N+1}=0$. Finally, for $x \in\{1, \ldots, N-1\}$,

$$
\begin{aligned}
p_{x}+q_{x+1}= & \frac{p(x / N)-x p(x / N)}{N} \\
& +\frac{(x+1)}{N}-\frac{(x+1) p((x+1) / N)}{N} \\
\leq & p\left(\frac{x}{N}\right)\left(\frac{1-x / N-(x+1)}{N}\right) \\
& +\frac{(x+1)}{N} \\
= & \frac{[p(x / N)(N-2 x-1)+x+1]}{N} \leq 1
\end{aligned}
$$

because

$$
\begin{gathered}
p\left(\frac{x}{N}\right) \leq \frac{(1-x /(N-1))}{(1-2 x /(N-1))}, \\
\text { if } \left.x \in\left\{0, \ldots, \mid \frac{N-1}{2}\right]\right\} \\
p\left(\frac{x}{N}\right) \geq \frac{(1-x /(N-1))}{(1-2 x /(N-1))}, \\
\text { if } x \in\left\{\left[\frac{N-1}{2}\right], \ldots, N-1\right\} .
\end{gathered}
$$

For the mutation Moran model in the weak mutation regime $0<\mu<1, p(u)$ is nondecreasing, and therefore, this chain is stochastically monotone. Although the condition that $p(u)$ is nondecreasing is a sufficient condition to guarantee that the chain is stochastically monotone, it is not necessary.

Proposition 12. Consider the Moran model $X_{n}$ with mutation bias $p$ in the strong mutation regime $2>\mu \geq 1$. Although $p(u)$ is not nondecreasing, the condition $p_{x}+q_{x+1} \leq 1$ is still fulfilled would $N$ be large enough (in which case the Moran chain with strong mutations is still stochastically monotone). And then, the Siegmund dual exists.

Proof. When $2>\mu>1, p(u)=\mu_{1}+\bar{\mu} u$ is monotone decreasing. We need to check that in that case also $p_{x}+q_{x+1} \leq$ 1 when $x$ varies from 0 to $N-1$, as soon as $N$ is large enough. After some elementary algebra,

$$
\begin{aligned}
p_{x}+q_{x+1}-1= & -2 \bar{\mu}\left(\frac{x}{N}\right)^{2} \\
& +\left(2 \bar{\mu}_{1}-\mu-2 \frac{\bar{\mu}}{N}\right)\left(\frac{x}{N}\right) \\
& -\left(\bar{\mu}_{1}-\frac{\bar{\mu}_{1}}{N}+\frac{\bar{\mu}}{N^{2}}\right) .
\end{aligned}
$$


The minimum if this convex degree- 2 polynomial is attained at the point $x=N\left(2 \bar{\mu}_{1}-\mu-2(\bar{\mu} / N)\right) /(4 \bar{\mu})$ so, when $N$ is large, to the left (right) of $x=N / 2$ if $\mu_{2}>\mu_{1}$ (if $\mu_{1}>\mu_{2}$ ) with a negative value of this minimum. The maximum of $p_{x}+$ $q_{x+1}-1$ is thus attained either at $x=N-1($ at $x=0)$, with $p_{N-1}+q_{N}-1=-\bar{\mu}_{2}+(1 / N)\left(\bar{\mu}_{2}-\bar{\mu} / N\right)$ (resp., $p_{0}+q_{1}-1=$ $\left.-\bar{\mu}_{1}+(1 / N)\left(\bar{\mu}_{1}-\bar{\mu} / N\right)\right)$. These maxima are nonpositive for all $N$ sufficiently large so that $N^{2}-N>\max \left(-\bar{\mu} / \bar{\mu}_{2},-\bar{\mu} / \bar{\mu}_{1}\right)$. Then, given a Moran model with $2>\mu>1$, for all $N$ large enough, both $p_{N-1}+q_{N}-1<0$ and $p_{0}+q_{1}-1<0$.

When $\mu=1, p(u)=\mu_{1}$ and $p_{x}+q_{x+1}$ is a linear function of $x$. The maximum of $p_{x}+q_{x+1}-1$ is thus attained either at $x=N-1($ at $x=0)$, with $p_{N-1}+q_{N}-1=-\left(\mu_{1} / N\right)(N-1)$ (resp., $\left.p_{0}+q_{1}-1=-\left(\bar{\mu}_{1} / N\right)(N-1)\right)$, both nonpositive. So $p_{x}+q_{x+1} \leq 1$ for all $x$ and the Siegmund dual also exists when $\mu=1$, whatever the value of $N$.

Remark 13. Whenever $0<\mu<2$, therefore, the Moran model with mutations is or can be made stochastically monotone. When $\mu=2, p_{x}+q_{x+1}-1<0$ except at least at both $x=0$ and $x=N-1$ where $p_{x}+q_{x+1}-1=1 / N^{2}>0$. The BernoulliLaplace chain fails to be stochastically monotone.

From the structure of $\widehat{P}$, it is apparent that the dual process loses mass at $y=0$ and is absorbed at $y=N$. Let us therefore add a coffin state $\partial:=\{-1\}$ and consider the enlarged stochastic matrix which we will call $\widehat{P}_{\partial}$ :

$$
\widehat{P}_{\partial}=\left[\begin{array}{cccccc}
1 & 0 & & & & \\
1-r_{0} & r_{0}-q_{1} & q_{1} & & & \\
& p_{1} & \widehat{r}_{1} & q_{2} & & \\
& & \ddots & \ddots & \ddots & \\
& & & p_{N-1} & \widehat{r}_{N-1} & q_{N} \\
& & & & 0 & 1
\end{array}\right] .
$$

The corresponding proper $\mathrm{BD}$ chain, call it ${ }_{\partial} \widehat{X}_{n}$, now has two absorbing states, one at $\{-1\}$ and one at $\{N\}$. Let now $\widehat{\varphi}(y), y=-1,0,1, \ldots, N$ be the scale function of ${ }_{\partial} \widehat{X}_{n}$, solving $\widehat{P}_{\partial} \widehat{\varphi}=\widehat{\varphi}$, forcing $\widehat{\varphi}(-1)=0$. We can easily check that

$$
\begin{gathered}
\widehat{\varphi}(-1)=0, \quad \widehat{\varphi}(0)=1, \\
\widehat{\varphi}(y)=\gamma_{y}^{c}:=\sum_{z=0}^{y} \gamma_{z}=\frac{1}{\pi_{0}} \sum_{z=0}^{y} \pi_{z}
\end{gathered}
$$

so that the scale function of ${ }_{\partial} \widehat{X}_{n}$ expresses in terms of the cumulative distribution $\pi_{y}^{c}:=\sum_{z=0}^{y} \pi_{z}$ of the invariant measure of the original process.

Let $\widehat{\tau}_{y}:=\widehat{\tau}_{y,-1} \wedge \widehat{\tau}_{y, N}$ be the infimum of the first hitting time of $\{-1\}$ and $\{N\}$ starting from $y \in\{0, \ldots, N-1\}$. We have

$$
\mathbf{P}_{y}\left({ }_{\partial} \widehat{X}_{\widehat{\tau}_{y}}=N\right)=\frac{\widehat{\varphi}(y)}{\widehat{\varphi}(N)}=\frac{\gamma_{y}^{c}}{\gamma_{N}^{c}}=\pi_{y}^{c} .
$$

Doob $h$-Transform. Define a new transition matrix $\widetilde{P}_{\partial}$ by

$$
\widetilde{P}_{\partial}(x, y)=\frac{\pi_{y}^{c}}{\pi_{x}^{c}} \widehat{P}_{\partial}(x, y), \quad x, y \in\{-1,0, \ldots, N\}^{2} .
$$

We have

$$
\widetilde{P}_{\partial}=\left[\begin{array}{cccccc}
1 & 0 & & & & \\
0 & r_{0}-q_{1} & p_{0}+q_{1} & & & \\
& \frac{\pi_{0}^{c}}{\pi_{1}^{c}} p_{1} & \widehat{r}_{1} & \frac{\pi_{2}^{c}}{\pi_{1}^{c}} q_{2} & & \\
& & \ddots & \ddots & \ddots & \\
& & & \frac{\pi_{N-2}^{c}}{\pi_{N-1}^{c}} p_{N-1} & \widehat{r}_{N-1} & \frac{\pi_{N}^{c}}{\pi_{N-1}^{c}} q_{N} \\
& & & & 0 & 1
\end{array}\right],
$$

where state $\{-1\}$ becomes isolated and disconnected. Deleting the row and column associated to the entry $\{-1\}$ of $\widetilde{P}_{\partial}$, we get a stochastic matrix, call it $\widetilde{P}$, of a process $\widetilde{X}_{n}$ on $\{0, \ldots, N\}$ which corresponds to ${ }_{\partial} \widehat{X}_{n}$ conditioned to first hit state $\{N\}$ before state $\{-1\}$. The state $\{0\}$ of this conditioned BD process now is partially reflecting whereas the remaining absorbing state, say $a$, is $a=\{N\}$.

We will also need subsequently to introduce $\tilde{\varphi}(x), x=$ $0,1, \ldots, N$, which is the new scale function of $\widetilde{X}_{n}$. We have

$$
\begin{gathered}
\tilde{\varphi}(0)=0, \quad \widehat{\varphi}(1)=1, \\
\widehat{\varphi}(x)=1+\sum_{y=1}^{x-1} \prod_{z=1}^{y} \frac{\widetilde{q}_{z}}{\widetilde{p}_{z}}=1+\sum_{y=1}^{x-1} \prod_{z=1}^{y} \frac{\pi_{z+1}^{c}}{\pi_{z-1}^{c}} \frac{q_{z+1}}{p_{z}}
\end{gathered}
$$

because $\tilde{q}_{z}=\left(\pi_{z+1}^{c} / \pi_{z}^{c}\right) q_{z+1}, \tilde{p}_{z}=\left(\pi_{z-1}^{c} / \pi_{z}^{c}\right) p_{z}$ and $\tilde{r}_{z}=$ $\widehat{r}_{z}, \quad z=1, \ldots, N-1, \widetilde{q}_{0}=p_{0}+q_{1}$ and $\widetilde{r}_{0}=r_{0}-q_{1}$ and $\widetilde{r}_{N}=1$ and $\widetilde{p}_{N}=0$ are the new transition probabilities of $\widetilde{X}_{n}$ on $\{0, \ldots, N\}$ which can now be read from $\widetilde{P}$.

We now show that $\widetilde{P}$ and $P$ are intertwined through a stochastic link.

Proposition 14. (i) The matrices $\widetilde{P}$ and $P$ are similar (with the same eigenvalues); that is,

$$
\widetilde{P}=\Lambda P \Lambda^{-1}
$$

The link $\Lambda$ is given by $\Lambda(\tilde{x}, x)=\left(\pi_{x} / \pi_{\tilde{x}}^{c}\right) \mathbf{1}(x \leq \tilde{x})$, corresponding to the entries of a lower-triangular stochastic matrix. In other words, for all $n \geq 0$,

$$
\Lambda(\tilde{x}, x)=\mathbf{P}\left(X_{n}=x \mid \widetilde{X}_{n}=\tilde{x}\right),
$$

and $\boldsymbol{\pi}_{n}^{\prime}=\tilde{\boldsymbol{\pi}}_{n}^{\prime} \Lambda$, where $\boldsymbol{\pi}_{n}=\mathbf{P}_{\boldsymbol{\pi}_{0}}\left(X_{n}=\cdot\right)$ and $\tilde{\boldsymbol{\pi}}_{n}=\mathbf{P}_{\widetilde{\pi}_{0}}\left(\widetilde{X}_{n}=\cdot\right)$.

(ii) The link $\Lambda$ satisfies

$$
\Lambda(N, x)=\pi_{x}, \quad x=0, \ldots, N .
$$

(iii) $\tilde{\boldsymbol{\pi}}_{0}^{\prime}=\boldsymbol{\pi}_{0}^{\prime}=\mathbf{e}_{0}^{\prime}:=(1,0, \ldots, 0)$ are admissible initial distributions of the chains $\widetilde{X}_{n}$ and $X_{n}$, satisfying

$$
\pi_{0}^{\prime}=\tilde{\pi}_{0}^{\prime} \Lambda \text {. }
$$

(iv) $\widetilde{P}$ is $K$-dual to $P$ :

$$
\widetilde{P}^{\prime}=K^{-1} P K,
$$

where $K(x, y)=\left(1 / \pi_{y}^{c}\right) \mathbf{1}(x \leq y)$. 
Proof. (i) From (120),

$$
\widetilde{P}=D_{\pi^{c}}^{-1} \widehat{P} D_{\pi^{c}}, \quad \text { where } D_{\pi^{c}}=\operatorname{diag}\left(\pi_{0}^{c}, \ldots, \pi_{N}^{c}\right) .
$$

The random walk $X_{n}$ being reversible, $P^{\prime}=D_{\pi} P D_{\pi}^{-1}$. Thus,

$$
\begin{aligned}
\widetilde{P} & =D_{\pi^{c}}^{-1} H^{\prime} P^{\prime} H^{\prime-1} D_{\pi^{c}} \\
& =D_{\pi^{c}}^{-1} H^{\prime} D_{\pi} P D_{\pi}^{-1} H^{\prime-1} D_{\pi^{c}} .
\end{aligned}
$$

As a result, $\Lambda=D_{\pi^{c}}^{-1} H^{\prime} D_{\pi}$ with the right entries $\Lambda(\tilde{x}, x)=$ $\left(\pi_{x} / \pi_{\tilde{x}}^{c}\right) \mathbf{1}(x \leq \tilde{x})$, satisfying $\sum_{x \leq \tilde{x}}\left(\pi_{x} / \pi_{\tilde{x}}^{c}\right)=1$.

(ii) The last row of $\Lambda$ is given by $\Lambda(N, x)=\pi_{x}$ so that once $\widetilde{X}_{n}$ hits state $N$, the law of $X_{n}$ is $\pi$.

(iii) The first row of $\Lambda$ is $(1,0, \ldots, 0)$ so that $\mathbf{e}_{0}^{\prime}=\mathbf{e}_{0}^{\prime} \Lambda$.

(iv) Indeed, $\widetilde{P}^{\prime}=D_{\pi^{c}} \widehat{P}^{\prime} D_{\pi^{c}}^{-1}=D_{\pi^{c}} H^{-1} P H D_{\pi^{c}}^{-1}:=K^{-1} P K$ with $K=H D_{\pi^{c}}^{-1}$ with the right entries.

So $\widetilde{P}$ (as the composition of the Siegmund dual of $P$ with a Doob-transform) can be obtained from $P$ either from (i) through a stochastic link $\Lambda$ or from (iv) through the duality kernel $K$. This is because BD chains such as the Moran model are reversible.

Strong Stationary Time. The intertwining construction shows that the original positive recurrent BD chain $X_{n}$ with transition matrix $P$ may also be viewed as the output (through the link $\Lambda$ ) of an intertwined hidden Markov chain $\widetilde{X}_{n}$ with transition matrix $\widetilde{P}$. Once $\widetilde{X}_{n}$ hits its absorbing state $\{N\}$, the RW $X_{n}$ is distributed like $\pi$, provided both $X_{n}$ and $\widetilde{X}_{n}$ were started at 0 . Furthermore, there exists a bivariate Markov chain $(\widetilde{X}, X)$ with transition kernel

$$
P((\tilde{x}, x),(\tilde{y}, y))=\frac{P(x, y) \cdot \widetilde{P}(\tilde{x}, \tilde{y}) \cdot \Lambda(\tilde{y}, y)}{(\Lambda P)(\tilde{x}, y)} \mathbf{1}_{(\Lambda P)(\tilde{x}, y)>0},
$$

where $\tilde{x} \in\{x, x \pm 1\}, \tilde{y} \in\{y, y \pm 1\}$. We have $(\Lambda P)(\tilde{x}, y)>0$ if and only if $y \leq \tilde{x}+1$. We can check that $\sum_{y} \sum_{\tilde{y}} P((\tilde{x}, x),(\tilde{y}, y))=1$ as required for a stochastic matrix.

With $x \in\{0, \ldots, N-1\}$, we will let

$$
\widetilde{\tau}_{\widetilde{x}_{0}, N}=\inf \left(n: \widetilde{X}_{n}=N \mid \widetilde{X}_{0}=\widetilde{x}_{0}\right)
$$

be the first hitting time of $\{N\}$ of $\widetilde{X}_{n}$, starting from state $\widetilde{x}_{0} \in$ $\{0, \ldots, N-1\}$. The random time $\widetilde{\tau}_{0, N}$ gives some information on the speed of convergence of the law of the original process $X_{n}$ to its invariant measure (is a strong stationary time in the sense of Diaconis and Fill [5]). The facts (123), (124), (125), and (126) indeed guarantee that $\widetilde{\tau}_{0, N}$ is a strong stationary time of $X_{n}$ in the sense that $X_{\widetilde{\tau}_{0, N}} \stackrel{d}{\sim} \pi$ and is independent of $\widetilde{\tau}_{0, N}$ (see [5, Theorems 2.4 and 2.17] or [6, Theorem 2.1]). Equivalently (see [4, Proposition 3.2]), it holds that

$$
\operatorname{sep}\left(\pi_{n}, \pi\right) \leq \mathbf{P}\left(\tilde{\tau}_{0, N}>n\right) \leq \frac{\mathbf{E}\left(\widetilde{\tau}_{0, N}\right)}{n},
$$

where $\pi_{n}(\cdot):=P^{n}(0, \cdot)$ is the law of $X_{n}$ started at $0, \pi$ its invariant measure. In (132), the separation discrepancy is defined by $\operatorname{sep}\left(\boldsymbol{\pi}_{n}, \boldsymbol{\pi}\right)=\sup _{y}\left[1-\pi_{n}(y) / \pi_{y}\right]$. It satisfies $\operatorname{sep}\left(\boldsymbol{\pi}_{n}, \boldsymbol{\pi}\right) \geq$ $\left\|\pi_{n}-\pi\right\|_{T V}$, where $\left\|\pi_{n}-\pi\right\|_{T V}=(1 / 2) \sum_{y}\left|\pi_{n}(y)-\pi_{y}\right|$ is the total variation distance between $\pi_{n}$ and $\pi$.

Furthermore, from (123), (126), there is a unique "witness" state say $d=N$ such that either $\tilde{\pi}_{n}(N)=0$ or $\tilde{\pi}_{n}(N)>$ $0 \Rightarrow \pi_{n}(d)=\tilde{\pi}_{n}(N) \pi_{d}>0$ showing that this random time is stochastically the smallest since the first inequality in (132) turns out to be an equality (see [5, Remark 2.39] and Proposition 15 below).

In the context of BD chains absorbed at $N$, the probability generating function (pgf) of $\widetilde{\tau}_{0, N} \geq N$ is $[6,28]$ :

$$
\mathbf{E}\left(z^{\widetilde{\tau}_{0, N}}\right)=\prod_{k=1}^{N} \frac{\left(1-t_{k}\right)_{z}}{1-t_{k} z}, \quad z \in[0,1]
$$

where $-1<t_{k}<+1, k=1, \ldots, N$ are the $N$ distinct eigenvalues of both $\widetilde{P}$ and $P$, avoiding $t_{0}=1$. The formula (133) also reads

$$
\mathbf{E}\left(z^{\widetilde{\tau}_{0, N}}\right)=\sum_{l=1}^{N} A_{l} \frac{z\left(1-t_{l}\right)}{1-t_{l} z}
$$

where

$$
\begin{gathered}
A_{l}=\prod_{k \neq l} \frac{1-t_{k}}{t_{l}-t_{k}} \\
\mathbf{P}\left(\tilde{\tau}_{0, N}>n\right)=\sum_{l=1}^{N} \prod_{k \neq l} \frac{1-t_{k}}{t_{l}-t_{k}} t_{l}^{n}, \quad n \geq N-1 .
\end{gathered}
$$

Thus, $t_{1}^{-n} \mathbf{P}\left(\widetilde{\tau}_{0, N}>n\right) \rightarrow{ }_{n \uparrow \infty} \prod_{k=2}^{N}\left(\left(1-t_{k}\right) /\left(t_{1}-t_{k}\right)\right)$, and $\tilde{\tau}_{0, N}$ has geometric tails with exponent $t_{1}$. We have

$$
\begin{aligned}
\mathbf{E}\left(\tilde{\tau}_{0, N}\right)= & \operatorname{trace}(I-\widetilde{P})^{-1}=\sum_{k=1}^{N}\left(1-t_{k}\right)^{-1}, \\
\sigma^{2}\left(\widetilde{\tau}_{0, N}\right) & =\sum_{k=1}^{N} t_{k}\left(1-t_{k}\right)^{-2} \\
& =\sum_{k=1}^{N}\left(1-t_{k}\right)^{-2}-\sum_{k=1}^{N}\left(1-t_{k}\right)^{-1} .
\end{aligned}
$$

Note that, since $t_{1}$ is the dominant eigenvalue,

$$
\sigma^{2}\left(\widetilde{\tau}_{0, N}\right) \leq \frac{\mathbf{E}\left(\widetilde{\tau}_{0, N}\right)}{1-t_{1}}
$$

When the eigenvalues $t_{k}$ are nonnegative, then $\widetilde{\tau}_{0, N} \stackrel{d}{=} \sum_{k=1}^{N} \tau_{k}$ where the $\tau_{k}$ s are independent with $\tau_{k} \stackrel{d}{\sim} \operatorname{geom}\left(1-t_{k}\right)$ the geometric distribution with success parameter $1-t_{k}$ on $\{1,2, \ldots\}$. When the eigenvalues $t_{k}$ are not all positive, it is not obvious that the previous expression (133) of $\mathbf{E}\left(z^{\widetilde{\tau}_{0, N}}\right)$ is indeed a pgf but it turns out that this is the case. Assuming 
$t_{N}<\cdots<t_{l+1}<0 \leq t_{l}<\cdots<t_{1}<t_{0}=1$, (133) takes on the interpretation of

$$
\widetilde{\tau}_{0, N}-\sum_{k=l+1}^{N} b_{k} \stackrel{d}{=} \sum_{k=1}^{l} \tau_{k}
$$

where $b_{k} \stackrel{d}{\sim}$ bernoulli $\left(1 /\left(1-t_{k}\right)\right), \tau_{k} \stackrel{d}{\sim}$ geom $\left(1-t_{k}\right)$, and $\tilde{\tau}_{0, N}$ are all mutually independent. We can summarize these results that should mainly be attributed to [4-7] as follows.

Proposition 15. Suppose a Siegmund dual exists for a finite state space ergodic BD chain $X_{n}$. Then, there exists a Markov chain $\widetilde{X}_{n}$, intertwined with $X_{n}$, with $\{N\}$ as an absorbing state and fully described in Proposition 14. The random time $\widetilde{\tau}_{0, N}$ is a fastest strong stationary time for $X_{n}$ whose law is characterized either by (133) or (136) involving the spectrum of either $P$ or $\widetilde{P}$, the transition matrices governing the two processes.

Remark 16. When the RW is symmetric, except for $t_{N}=-1$ and maybe $\{0\}$, the eigenvalues can be paired, because if $t_{k} \epsilon$ $(0,1)$ is an eigenvalue, then $t_{N-k}=-t_{k}$ is one also. The pgf reads

$$
\begin{gathered}
\mathbf{E}\left(z^{\widetilde{\tau}_{0, N}}\right)=\frac{2 z^{2}}{1+z} \prod_{k=1}^{N_{0}-1} \frac{\left(1-t_{k}^{2}\right) z^{2}}{1-t_{k}^{2} z^{2}}, \quad \text { if } N=2 N_{0}, \\
\mathbf{E}\left(z^{\widetilde{\tau}_{0, N}}\right)=\frac{2 z}{1+z} \prod_{k=1}^{N_{0}} \frac{\left(1-t_{k}^{2}\right) z^{2}}{1-t_{k}^{2} z^{2}}, \quad \text { if } N=2 N_{0}+1,
\end{gathered}
$$

depending on whether $\{0\}$ is or is not an eigenvalue.

Computing the Mean and Variance of $\tilde{\tau}_{0, N}$. Suppose that the $t_{k}$ are known explicitly. In this case, it is possible to compute $\mathbf{E}\left(\widetilde{\tau}_{0, N}\right)$ and $\sigma^{2}\left(\widetilde{\tau}_{0, N}\right)$ and find conditions under which

$$
\begin{gathered}
\mathbf{E}\left(\widetilde{\tau}_{0, N}\right) \longrightarrow \infty, \\
\sigma^{2}\left(\frac{\widetilde{\tau}_{0, N}}{\mathbf{E}\left(\tilde{\tau}_{0, N}\right)}\right) \longrightarrow 0, \quad \text { as } N \uparrow \infty .
\end{gathered}
$$

If this is the case, then $\widetilde{\tau}_{0, N} / \mathbf{E}\left(\widetilde{\tau}_{0, N}\right) \rightarrow 1$ in probability and $\left\lfloor\mathbf{E}\left(\widetilde{\tau}_{0, N}\right) / 2\right\rfloor$ is expected to be a cut-off time for $X_{n}$ started at $\{0\}$.

We now apply this construction to the particular Moran model under study.

The Case of the Moran Model with Mutations. The eigenvalues $t_{k}$ are known, leading to: $1-t_{k}=(k / N)(\mu+\bar{\mu}((k-1) / N))$. If we scale the characteristic time $\widetilde{\tau}_{0, N}$ to $N$, the summations for large $N$ can be replaced by integrals, leading to the estimate

$$
\begin{aligned}
\mu_{N} \sim N \int_{0}^{1} \frac{d x}{(x+1 / N)(\mu+\bar{\mu} x)} \\
\quad=\frac{N^{2}}{N \mu-\bar{\mu}}\left(\int_{0}^{1} \frac{d x}{x+1 / N}-\bar{\mu} \int_{0}^{1} \frac{d x}{\mu+\bar{\mu} x}\right)
\end{aligned}
$$

and similarly for $\sigma^{2}\left(\widetilde{\tau}_{0, N}\right)$. From this, we easily get the following proposition.
Proposition 17. For the Moran model with mutations with $0<\mu<2$, whenever the Siegmund dual exists,

$$
\begin{gathered}
\mu_{N} \sim \frac{N}{\mu}(\log N+\log \mu), \\
\sigma^{2}\left(\widetilde{\tau}_{0, N}\right) \sim\left(\frac{N}{\mu}\right)^{2},
\end{gathered}
$$

showing that $\sigma^{2}\left(\widetilde{\tau}_{0, N} / \mathbf{E}\left(\widetilde{\tau}_{0, N}\right)\right) \sim(\log N)^{-2} \rightarrow 0$.

We have

$$
A_{l}=\prod_{k \neq l} \frac{1-t_{k}}{t_{l}-t_{k}}=\left(\prod_{k \neq l}\left(1-\frac{l(N \mu+(l-1) \bar{\mu})}{k(N \mu+(k-1) \bar{\mu})}\right)\right)^{-1},
$$

and the tail distribution of $\widetilde{\tau}_{0, N}$ is

$$
\mathbf{P}\left(\tilde{\tau}_{0, N}>n\right)=\sum_{l=1}^{N} \prod_{k \neq l} \frac{1-t_{k}}{t_{l}-t_{k}} t_{l}^{n}, \quad n \geq N-1 .
$$

Remark 18. When $\mu=2$, the Bernoulli-Laplace RW fails to be stochastically monotone, and so the above construction also fails as the chain has no Siegmund dual. However, as was shown in [29], similar conclusions on the fastest strong stationary time hold for the embedded continuoustime version of the Bernoulli-Laplace chain.

Proposition 19. We have the Gumbel limit law

$$
\widetilde{\tau}_{0, N}^{\text {Scaled }}:=\frac{\widetilde{\tau}_{0, N}-(N / \mu) \log N}{N / \mu} \stackrel{d}{\longrightarrow} X \stackrel{d}{\sim} e^{-\left(x+e^{-x}\right)}, \quad x \in \mathbb{R} .
$$

Proof. Indeed, using $t_{k} /\left(1-t_{k}\right) \sim N /(k \mu)$ to the dominant order in $N$,

$$
\begin{aligned}
& \mathbf{E}\left(e^{i t \widetilde{\tau}_{0, N}^{\text {scaled }}}\right) \\
& \sim N^{-i t} \prod_{k=1}^{N} \frac{1-t_{k}}{1-t_{k}(1+i t \mu / N)} \\
& \sim N^{-i t} \prod_{k=1}^{N} \frac{k}{k-i t} \longrightarrow \Gamma(1-i t),
\end{aligned}
$$

$$
\text { as } N \uparrow \infty \text {, }
$$

which is the Fourier transform of $e^{-\left(x+e^{-x}\right)}, x \in \mathbb{R}$.

$$
\begin{aligned}
& \text { With } n_{N}(\theta)=\lfloor(N / 2 \mu)(\log N+\theta)\rfloor \text {, then } \\
& \qquad\left\|P^{n_{N}(\theta)}(0, \cdot)-\pi\right\|_{T V} \underset{N \uparrow \infty}{\longrightarrow} c(\theta),
\end{aligned}
$$

where $c(\theta) \rightarrow_{\theta \uparrow \infty} 0$ and $c(\theta) \rightarrow_{\theta \uparrow-\infty} 1$. The expected mixing time is $\mu_{N} \sim(N / \mu) \log N$, whereas the spectral gap is $1-t_{1}=$ $\mu / N$, the product of the 2 of which tends to $\infty$. Recalling $\sigma^{2}\left(\widetilde{\tau}_{0, N}\right) \leq \mu_{N} /\left(1-t_{1}\right), \sigma^{2}\left(\tilde{\tau}_{0, N} / \mu_{N}\right)=\mu_{N}^{-2} \sigma^{2}\left(\widetilde{\tau}_{0, N}\right) \leq$ $1 /\left(\left(1-t_{1}\right) \mu_{N}\right)$, the condition $\left(1-t_{1}\right) \mu_{N} \rightarrow \infty$ is a sufficient condition for $\sigma^{2}\left(\widetilde{\tau}_{0, N} / \mu_{N}\right) \rightarrow 0$. If this holds, the contribution of $\sum_{k=2}^{N}\left(1-t_{k}\right)^{-1}$ to $\mu_{N}$ dominates the lead term $\left(1-t_{1}\right)^{-1}$ (see $[30,31]$ for recent developments and precisions). 


\section{Related Transition Times for the Moran Model with Mutations}

Consider the ergodic Moran birth and death Markov chains $X_{n}$ with mutations on the state space $\{0, \ldots, N\}$. By (16), the mean return time to state $\{0\}$ is $\mathbf{E}\left(\tau_{0,0}^{*}\right)=1 / \pi_{0}$. Thus, with $\rho=\left(\bar{\mu}_{1}^{\bar{\mu}_{1}} \mu^{\mu} / \mu_{2}^{\mu_{2}}\right)^{1 / \bar{\mu}}<1$, regardless of $\mu<1$ or $2>\mu>1$

$$
\mathbf{E}\left(\tau_{0,0}^{*}\right) \sim \sqrt{\frac{\mu \bar{\mu}_{1}}{\mu_{2}}}\left(\frac{1}{\rho}\right)^{N}, \quad \text { as } N \longrightarrow \infty .
$$

When $\mu=1, \mathbf{E}\left(\tau_{0,0}^{*}\right)=\bar{\mu}_{1}^{-N}=\mu_{2}^{-N}\left(=2^{N}\right.$ if $\left.\mu_{1}=\mu_{2}=1 / 2\right)$.

If $\mu=2\left(\mu_{1}=\mu_{2}=1\right), \mathbf{E}\left(\tau_{0,0}^{*}\right)=1 / \pi_{0}=\left(\begin{array}{c}2 N \\ N\end{array}\right) \sim 4^{N} / \sqrt{\pi N}$.

We wish now to estimate the expected time it takes for $X_{n}$ to move from one end of the state space to the other, that is, from state $\{0\}$ to $\{N\}$. Let $\tau_{0, N}$ be the random time of such a sweep (note that the expected fixation time of a wild-type allele is given by $\mathbf{E}\left(\tau_{1, N}\right)=\mathbf{E}\left(\tau_{0, N}\right)-1 / p_{0}$ as a result of $\tau_{0, N} \stackrel{d}{=} \tau_{1, N}+G$, the sum of $\tau_{1, N}$ and an independent geometrically distributed $\operatorname{rv} G \geq 1$ with success probability $\left.p_{0}\right)$. We will prove the following estimation of its mean value growing geometrically fast.

Proposition 20. With $\bar{\rho}=\left(\bar{\mu}_{2}^{\bar{\mu}_{2}} \mu^{\mu} / \mu_{1}^{\mu_{1}}\right)^{1 / \bar{\mu}}<1$, regardless of $\mu<1$ or $2>\mu>1$, it holds that

$$
\mathbf{E}\left(\tau_{0, N}\right) \sim \frac{1}{\mu_{2}} \sqrt{\frac{\mu \bar{\mu}_{2}}{\mu_{1}}}\left(\frac{1}{\bar{\rho}}\right)^{N}, \quad \text { as } N \longrightarrow \infty .
$$

When $\mu=1, \mathbf{E}\left(\tau_{0, N}\right) \sim \mu_{1}^{-N} / \mu_{2}\left(=2^{N+1}\right.$ in the lazy Ehrenfest case: $\left.\mu_{1}=\mu_{2}=1 / 2\right)$. When $\mu=2, \mathbf{E}\left(\tau_{0, N}\right) \sim 4^{N} / \sqrt{\pi N}$.

Proof. Let indeed $\tau_{x, x+1}$ be the random time to first hit the state $\{x+1\}$ starting from the state $\{x\}$. Depending on whether the move starting in $x$ is up, down, or no move, $\tau_{x, x+1}$ is either 1 or $1+\tau_{x, x+1}^{\prime}$ (with $\tau_{x, x+1}^{\prime}$ a statistical copy of $\tau_{x, x+1}$ ) or $1+$ $\tau_{x-1, x}+\tau_{x, x+1}^{\prime}$. If we let $\mu_{x}$ be the mean value of $\tau_{x, x+1}$, we thus get $\mu_{x}=p_{x}+r_{x}\left(1+\mu_{x}\right)+q_{x}\left(1+\mu_{x-1}+\mu_{x}\right), \quad x \geq 2$. This leads to the recurrence $\left(\mu_{0}=1 / p_{0}\right)$ :

$$
\mu_{x}=\frac{q_{x}}{p_{x}} \mu_{x-1}+\frac{1}{p_{x}}, \quad x \geq 1 .
$$

This recurrence can be solved explicitly to give

$$
\mu_{x}=\frac{1}{p_{x} \pi_{x}} \sum_{y=0}^{x} \pi_{y}=: \frac{\pi_{x}^{c}}{p_{x} \pi_{x}} .
$$

Thus,

$$
\begin{aligned}
\mathbf{E}\left(\tau_{0, N}\right) & =\sum_{x=0}^{N-1} \mu_{x}=\sum_{x=0}^{N-1} \frac{1}{p_{x} \pi_{x}} \sum_{y=0}^{x} \pi_{y} \\
& =\sum_{x=0}^{N-1} \frac{1}{p_{x}} \sum_{y=0}^{x} \prod_{z=y}^{x-1} \frac{q_{z+1}}{p_{z}} .
\end{aligned}
$$

Looking at this sum formula, one expects that its leading term is $\left(1 / p_{N-1} \pi_{N-1}\right) \sum_{y=0}^{N-1} \pi_{y}$ because this is where $\sum_{y=0}^{x} \pi_{y}$ is the largest and $\pi_{x}$ the smallest. Observing $\pi_{N-1} p_{N-1}=\pi_{N} q_{N}$ and $q_{N}=\mu_{2}, \sum_{y=0}^{N-1} \pi_{y}=1-\pi_{N} \rightarrow 1$, this estimation leads, recalling $\pi_{N} \sim \sqrt{\mu_{1} / \mu \bar{\mu}_{2}} \bar{\rho}^{N}$, to

$$
\mathbf{E}\left(\tau_{0, N}\right) \sim \frac{1}{p_{N-1} \pi_{N-1}} \sim \frac{1}{\mu_{2} \pi_{N}} \sim \frac{1}{\mu_{2}} \sqrt{\frac{\mu \bar{\mu}_{2}}{\mu_{1}}}\left(\frac{1}{\bar{\rho}}\right)^{N},
$$

and this is indeed true because the remaining terms do not contribute significantly, see [32]. When $\mu=1$,

$$
\mathbf{E}\left(\tau_{0, N}\right) \sim \frac{1}{\mu_{2} \pi_{N}}=\frac{\mu_{1}^{-N}}{\mu_{2}} .
$$

When $\mu=2$ (Laplace-Bernoulli), $\mathbf{E}\left(\tau_{0, N}\right) \sim 1 / \mu_{2} \pi_{N}=\left(\begin{array}{c}2 N \\ N\end{array}\right)$.

Remark 21. Regardless of whether $\mu \leq 1$ or $2>\mu>1, \rho>\bar{\rho}$ if and only if $\mu_{2}>\mu_{1}$. In this case, the limiting growth rate of the expected value of $\tau_{0, N}$ dominates the one of $\tau_{0,0}^{*}$. On the contrary, $\mu_{1}>\mu_{2} \Rightarrow \rho<\bar{\rho}$.

Note that if $\mu_{1}=\mu_{2}=\mu / 2$ (symmetric Moran model), $\rho=\bar{\rho}$ and $\left(\mathbf{E}\left(\tau_{0, N}\right) / \mathbf{E}\left(\tau_{0,0}^{*}\right)\right)^{1 / N} \rightarrow 1$ as $N \rightarrow \infty$, whereas $\mathbf{E}\left(\tau_{0, N}\right) / \mathbf{E}\left(\tau_{0,0}^{*}\right) \rightarrow 2 / \mu(>1$ if $\mu<2,=1$ if $\mu=2)$.

The physical image of the situation is the following: it takes a small $\mathcal{O}(N)$ amount of time to move from $\{0\}$ to the stable equilibrium state $\left\{N u_{*}\right\}$ because the drift is favorable to this displacement. Once at state $\left\{N u_{*}\right\}, X_{n}$ returns rapidly and very often to $\left\{N u_{*}\right\}$ (it takes a $\mathcal{O}(\sqrt{N})$ time to do so), and coming back either to $\{0\}$ or to $\{N\}$ is hard because the drift now pushes $X_{n}$ inside the domain $\{0, \ldots, N\} \ldots$ As a result, both $\mathbf{E}\left(\tau_{0, N}\right)$ and $\mathbf{E}\left(\tau_{0,0}^{*}\right)$ are, comparatively, geometrically very large, with rates computed just before. Recalling it takes about $(N \log N) / \mu$ steps for the chain to reach maximum entropy under the "sep-distance," starting from $\{0\}$, although the return to $\{0\}$ will eventually occur, the time it takes is so huge that such returns will not be observed. In other words, the chain will reach rapidly the invariant measure inside a single excursion. Let us finally check these last statements.

First Return Time to Equilibrium State. Indeed, from (75), using (86),

$$
\pi_{N u_{*}}=\frac{\left(\begin{array}{c}
-N v_{1} \\
N u_{*}
\end{array}\right)\left(\begin{array}{c}
-N v_{2} \\
N\left(1-u_{*}\right.
\end{array}\right)}{\left(\begin{array}{c}
-N \nu \\
N
\end{array}\right)} \sim \frac{1}{\sqrt{2 \pi \mu N}} .
$$

Thus, by (16), $\mathbf{E}\left(\tau_{N u_{*}, N u_{*}}^{*}\right) \sim \sqrt{2 \pi \mu N}=\mathcal{O}(\sqrt{N})$, and the return time to the equilibrium state is relatively small.

First Hitting Time of the Equilibrium State, Starting from $\{0\}$. In this case, we have

$$
\mathbf{E}\left(\tau_{0, N u_{*}}\right)=\sum_{x=0}^{N u_{*}-1} \frac{1}{p_{x}} \sum_{y=0}^{x} \prod_{z=y}^{x-1} \frac{q_{z+1}}{p_{z}} .
$$


If we scale this characteristic time to $N$, the summations for large $N$ can be replaced by integrals, leading to the estimate

$$
\mathbf{E}\left(\tau_{0, N u_{*}}\right) \sim N^{2} \int_{0}^{u_{*}} \frac{d v}{(1-v)\left(\mu_{1}+\bar{\mu} v\right)} \int_{0}^{v} d u e^{N(V(u)-V(v))} .
$$

Recalling

$$
\int_{0}^{v} d u e^{N V(u)} \sim e^{N V\left(u_{*}\right)} \sqrt{\frac{\pi}{2 N\left|V^{\prime \prime}\left(u_{*}\right)\right|}}, \quad \text { if } v \leq u_{*},
$$

two successive appeals to the saddle point method show that $\mathbf{E}\left(\tau_{0, N u_{*}}\right) \sim\left(\pi N^{2} / 2 N\left|V^{\prime \prime}\left(u_{*}\right)\right|\right)\left(\mu^{2} / \mu_{1} \mu_{2}\right)=(\pi / 2 \mu) N$, which is indeed $\mathcal{O}(N)$.

Concerning the related first hitting time of state $N u_{*}$, starting from $N u_{*}-1$ (one step below), we have

$$
\begin{aligned}
\mathbf{E}\left(\tau_{N u_{*}-1, N u_{*}}\right) & \sim \frac{N \mu^{2}}{\mu_{1} \mu_{2}} \int_{0}^{u_{*}} d u e^{N\left(V(u)-V\left(u_{*}\right)\right)} \\
& \sim \frac{\mu^{2}}{\mu_{1} \mu_{2}} \sqrt{\frac{\pi N}{2\left|V^{\prime \prime}\left(u_{*}\right)\right|}} \\
& =\sqrt{\frac{\pi \mu N}{2 \mu_{1} \mu_{2}}},
\end{aligned}
$$

showing that $\mathbf{E}\left(\tau_{N u_{*}-1, N u_{*}}\right)$ is also $\mathcal{O}(\sqrt{N})$, in accordance with $\mathbf{E}\left(\tau_{N u_{*}, N u_{*}}^{*}\right)=\mathcal{O}(\sqrt{N})$.

\section{Acknowledgments}

Thierry E. Huillet acknowledges partial support from the ANR Modélisation Aléatoire en Écologie, Génétique et Évolution (ANR-Manège-09-BLAN-0215 project) and from the MME-DII Center of Excellence (Modèles Mathématiques et Économiques de la Dynamique, de l'Incertitude et des Intéractions, ANR-11-LABX-0023-01 project).

\section{References}

[1] P. Flajolet and T. Huillet, "Analytic combinatorics of the Mabinogion urn," in Proceedings of the 5th Colloquium on Mathematics and Computer Science, Discrete Mathematics \& Theoretical Computer Science Proceedings Association for Artificial Intelligence, pp. 549-571, Discrete Mathematics \& Theoretical Computer Science, 2008.

[2] U. Garibaldi and P. Viarengo, "Exchangeability and non-selfaveraging," Journal of Economic Interaction and Coordination, vol. 7, no. 2, pp. 181-195, 2012.

[3] P. A. P. Moran, "Random processes in genetics," Proceedings of the Cambridge Philosophical Society, vol. 54, pp. 60-71, 1958.

[4] D. Aldous and P. Diaconis, "Strong uniform times and finite random walks," Advances in Applied Mathematics, vol. 8, no. 1, pp. 69-97, 1987.

[5] P. Diaconis and J. A. Fill, "Strong stationary times via a new form of duality, Annals of Probability, vol. 18, no. 4, pp. 1483-1522, 1990.
[6] J. A. Fill, "The passage time distribution for a birth-and-death chain: strong stationary duality gives a first stochastic proof," Journal of Theoretical Probability, vol. 22, no. 3, pp. 543-557, 2009.

[7] J. A. Fill, "On hitting times and fastest strong stationary times for skip-free and more general chains," Journal of Theoretical Probability, vol. 22, no. 3, pp. 587-600, 2009.

[8] S. Karlin and J. McGregor, "Random walks," Illinois Journal of Mathematics, vol. 3, pp. 66-81, 1959.

[9] W. Feller, An Introduction to Probability Theory and Its Applications, vol. 1, John Wiley and Sons, New York, NY, USA, 3rd edition, 1968.

[10] F. R. Gantmacher, “Tome 2: questions spéciales et applications," in Théorie des Matrices, vol. 19 of Collection Universitaire de Mathématiques, Traduit du Russe par Ch. Sarthou, Dunod, Paris, 1966.

[11] T. Whitehurst, "An application of orthogonal polynomials to random walks," Pacific Journal of Mathematics, vol. 99, no. 1, pp. 205-213, 1982.

[12] T. Maruyama, Stochastic Problems in Population Genetics, vol. 17 of Lecture Notes in Biomathematics, Springer, Berlin, Germany, 1977.

[13] J. H. Gillespie, The Causes of Molecular Evolution, Oxford University Press, Oxford, UK, 1991.

[14] W. J. Ewens, Mathematical Population Genetics. I. Theoretical Introduction, vol. 27 of Interdisciplinary Applied Mathematics, Springer, New York, NY, USA, 2nd edition, 2004.

[15] T. Huillet and M. Möhle, "On the extended Moran model and its relation to coalescents with multiple collisions," Theoretical Population Biology, 2012.

[16] T. Mansour, "Combinatorial identities and inverse binomial coefficients," Advances in Applied Mathematics, vol. 28, no. 2, pp. 196-202, 2002.

[17] G. Sella and A. E. Hirsh, "The application of statistical physics to evolutionary biology," Proceedings of the National Academy of Sciences of the United States of America, vol. 102, no. 27, pp. 9541-9546, 2005.

[18] S. Karlin and J. McGregor, "The Hahn polynomials, formulas and an application," Scripta Mathematica, vol. 26, pp. 33-46, 1961.

[19] S. Karlin and J. McGregor, "On a genetics model of Moran," Proceedings of the Cambridge Philosophical Society. Cambridge Philosophical Society, vol. 58, pp. 299-311, 1962.

[20] F. A. Grünbaum, "Random walks and orthogonal polynomials: some challenges," in Probability, Geometry and Integrable Systems, vol. 55 of Mathematical Sciences Research Institute Publications, pp. 241-260, Cambridge University Press, Cambridge, UK, 2008.

[21] E. Seneta, "Characterization by orthogonal polynomial systems of finite Markov chains. Probability, statistics and seismology," Journal of Applied Probability A, vol. 38, pp. 42-52, 2001.

[22] Y. Horibe, "On the increase of conditional entropy in Markov chains," in Transactions of the Tenth Prague Conference on Information Theory, Statistical Decision Functions, Random Processes A, pp. 391-396, Prague, Czech Republic, 1988, Reidel, Dordrecht, The Netherlands, 1988.

[23] S. R. S. Varadhan, "Large deviations," Annals of Probability, vol. 36, no. 2, pp. 397-419, 2008.

[24] T. M. Liggett, Interacting Particle Systems, vol. 276 of Grundlehren der Mathematischen Wissenschaften [Fundamental Principles of Mathematical Sciences], Springer, New York, NY, USA, 1985. 
[25] T. E. Huillet, "A duality approach to the genealogies of discrete nonneutral Wright-Fisher models," Journal of Probability and Statistics, vol. 2009, Article ID 714701, 22 pages, 2009.

[26] T. Huillet and M. Möhle, "Duality and asymptotics for a class of nonneutral discrete moran models," Journal of Applied Probability, vol. 46, no. 3, pp. 866-893, 2009.

[27] D. Siegmund, "The equivalence of absorbing and reflecting barrier problems for stochastically monotone Markov processes," Annals of Probability, vol. 4, no. 6, pp. 914-924, 1976.

[28] J. Keilson, Markov Chain Models-Rarity and Exponentiality, vol. 28 of Mathematical Sciences, Springer, New York, NY, USA, 1979.

[29] P. Diaconis and M. Shahshahani, "Time to reach stationarity in the Bernoulli-Laplace diffusion model," SIAM Journal on Mathematical Analysis, vol. 18, no. 1, pp. 208-218, 1987.

[30] P. Diaconis, "The cutoff phenomenon in finite Markov chains," Proceedings of the National Academy of Sciences of the United States of America, vol. 93, no. 4, pp. 1659-1664, 1996.

[31] P. Diaconis and L. Saloff-Coste, "Separation cut-offs for birth and death chains," Annals of Applied Probability, vol. 16, no. 4, pp. 2098-2122, 2006.

[32] B. Dunham, "Fluctuation theory for Moran's genetics model," Journal of Mathematical Analysis and Applications, vol. 210, no. 2, pp. 777-789, 1997. 


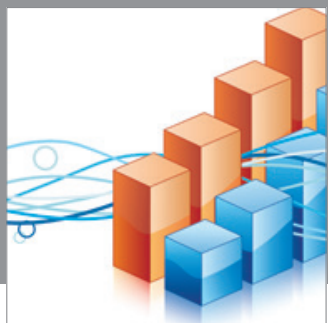

Advances in

Operations Research

mansans

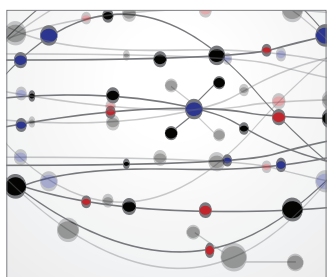

The Scientific World Journal
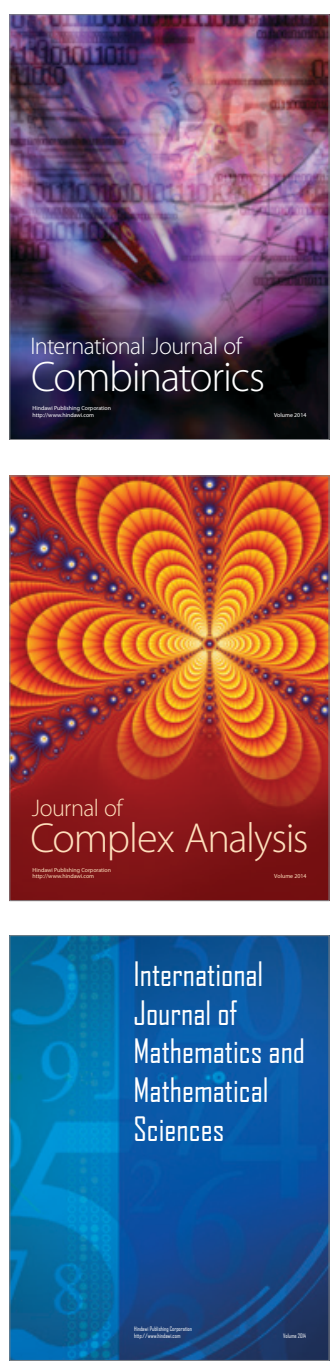
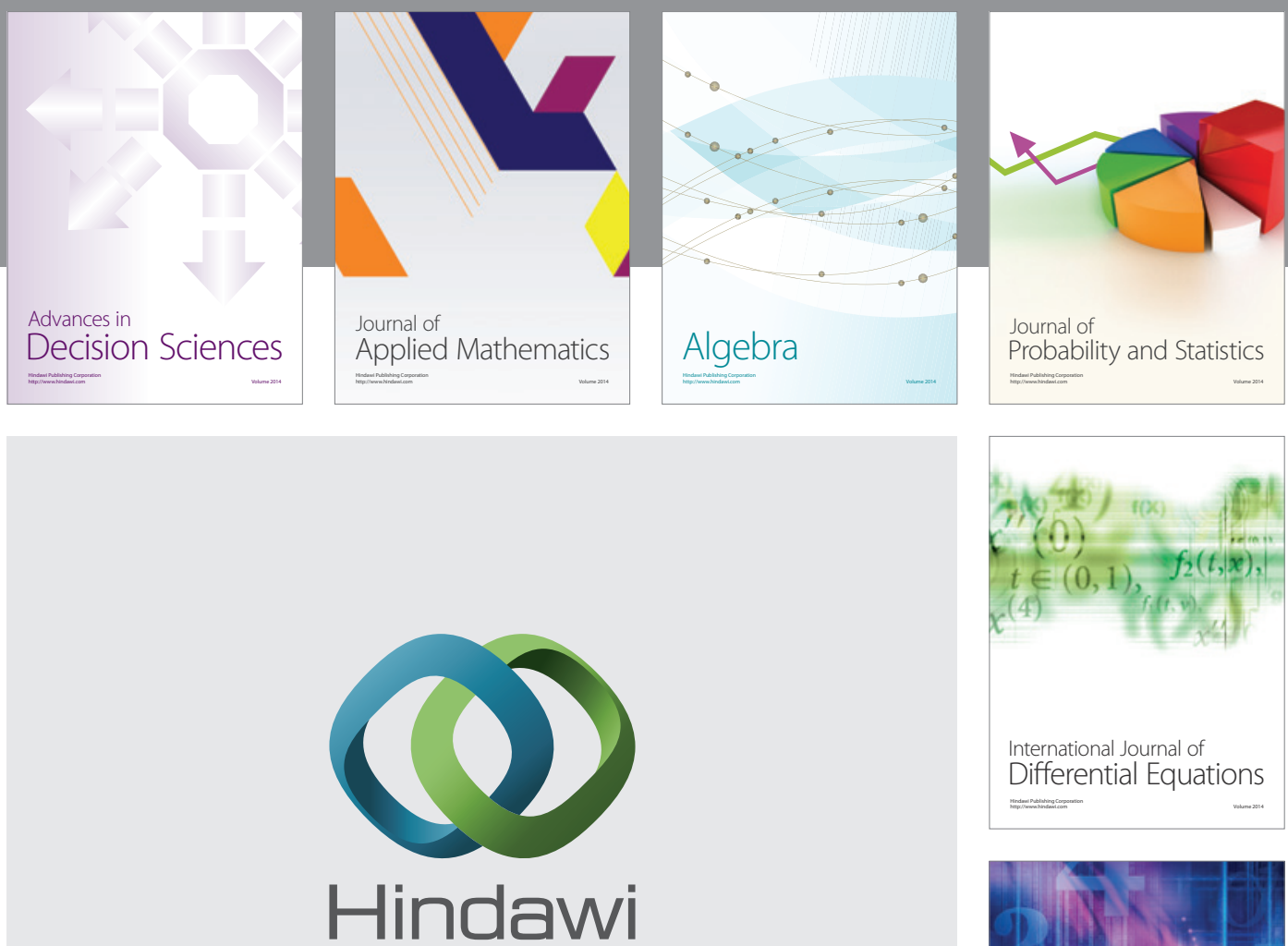

Submit your manuscripts at http://www.hindawi.com
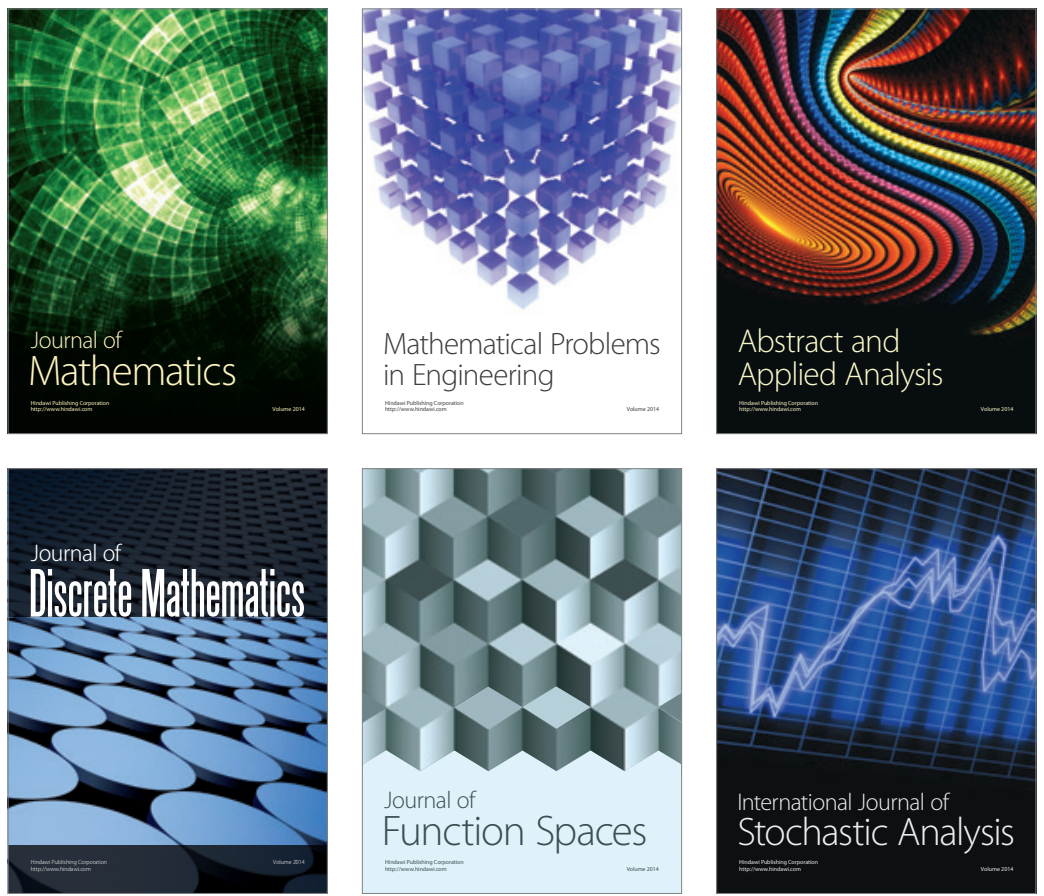

Journal of

Function Spaces

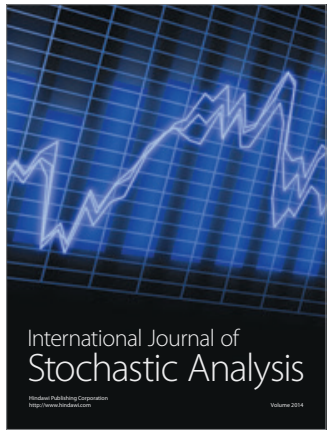

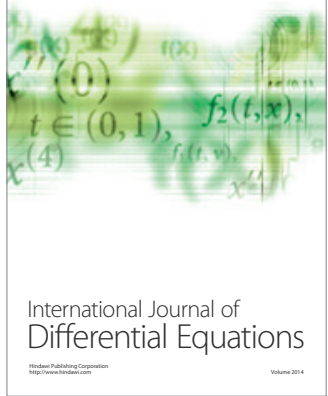
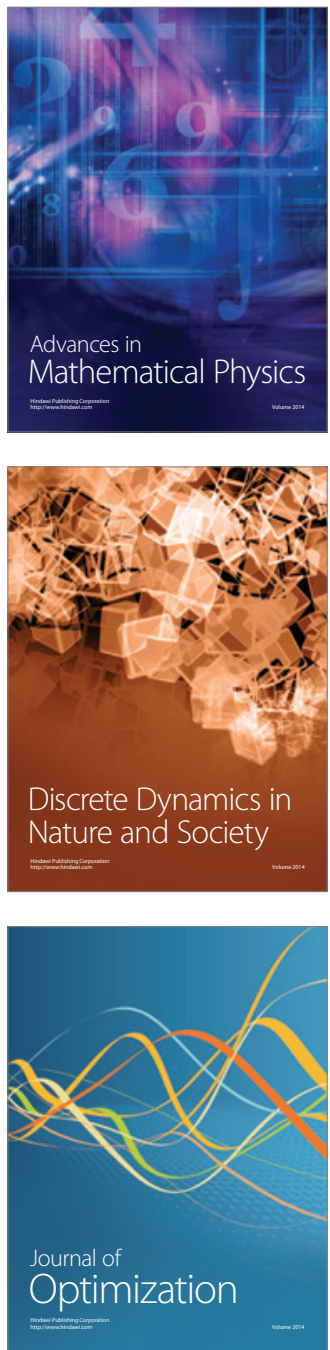